\title{
A Dynamic Network Simulation of the Nematode Tap Withdrawal Circuit: Predictions Concerning Synaptic Function Using Behavioral Criteria
}

\author{
Stephen R. Wicks, ${ }^{1}$ Chris J. Roehrig, ${ }^{1}$ and Catharine H. Rankin ${ }^{2}$ \\ 1 Program in Neuroscience and '2Department of Psychology, University of British Columbia, Vancouver, British Columbia, \\ Canada V6T $1 Z 4$
}

The nematode tap withdrawal reflex demonstrates several forms of behavioral plasticity. Although the neural connectivity that supports this behavior is identified (Integration of mechanosensory stimuli in Caenorhabditis elegans, Wicks and Rankin, 1995, J Neurosci 15:2434-2444), the neurotransmitter phenotypes, and hence whether the synapses in the circuit are excitatory or inhibitory, remain uncharacterized. Here we use a novel strategy to predict the polarity configuration, i.e., the array of excitatory and inhibitory connections, of the nematode tap withdrawal circuit using an anatomically and physiologically justifiable dynamic network simulation of that circuit. The output of the modeled circuit was optimized to the behavior of animals, which possessed cir- cuits altered by surgical ablation by exhaustively enumerating an array of synaptic signs that constituted the modeled circuit. All possible polarity configurations were then compared, and a statistical analysis was used to determine whether, for a given synaptic class, a particular polarity was associated with a good fit to behavioral data. The results from four related experiments were used to predict the polarities of seven of the nine cell classes of the tap withdrawal circuit. In addition, the model was used to assess possible roles for two novel mechanosensory integration neurons: DVA and PVD.

Key words: Caenorhabditis elegans; mechanosensory; habituation; lesion; inhibition; reflex
Reflexes have long been recognized as the substrate of many aspects of organismal behavior in diverse phyla from invertebrates to mammals, including primates (Sherrington, 1906; Carew et al., 1972; du Lac et al., 1995). Reflexive behaviors are often produced by small neural architectures that are amenable to empirical and computational investigation (Selverston, 1985). Thus, reflexes can be used to delineate the mechanisms underlying various forms of behavioral plasticity, such as adaptation (Corfas and Dudai, 1990), inhibition (Rankin, 1991; Fischer and Carew, 1993; Vu et al., 1993), habituation (Groves et al., 1969; Krasne, 1969; Carew et al., 1972; Wicks and Rankin, 1995b), and associative conditioning (Kandel and Schwartz, 1982; Steinmetz et al., 1992). One system that is complex enough to exhibit sophisticated behaviors yet simple enough to accommodate a precise neural model of a reflexive behavior is the nematode Caenorhabditis elegans.

The nematode $C$. elegans has been the subject of intensive biological study over the past three decades (Brenner, 1974; Wood, 1988) and has become a model system within which the relationship between genetics and behavior has been extensively studied. The adult nematode possesses only 302 neurons, each completely described in terms of location and morphology (Ward et al., 1975; Ware et al., 1975; White et al., 1986; Hall and Russell, 1991). The synaptic connectivity of the approximately 5000 chemical synapses, 600 gap junctions, and 2000 neuromuscular junc-

Received Oct. 16, 1995; revised March 22, 1996; accepted March 29, 1996.

This work was supported by a Natural Science and Engineering Research Council of Canada (NSERC) scholarship (S.R.W.), a British Columbia Science Council and NSERC awards (C.J.R.), and NSERC and Human Frontiers of Science operating grants (C.H.R.). We acknowledge fruitful discussions with D. M. Wilkie and B. Hutcheon during the inception of this work.

Correspondence should be addressed to Dr. Catharine H. Rankin, Department of Psychology, 2136 West Mall, University of British Columbia, Vancouver, British Columbia, Canada V6T $1 Z 4$.

Copyright $(\subset) 1996$ Society for Neuroscience $0270-6474 / 96 / 164017-15 \$ 05.00 / 0$ tions has been encoded in machine-readable form (Achacoso and Yamamoto, 1992). The extensive genetic analysis of the worm has confirmed that a number of basic biological mechanisms, such as second-messenger signaling (Gross et al., 1990; Lu et al., 1990; Mendel et al., 1995; Segalat et al., 1995), synaptic release (Nonet et al., 1993), and other cell-signaling events (Stern and DeVore, 1994), are conserved between it and more complex neurobiological systems. These advantages have been exploited by modeling researchers; chemotaxic (Lockery et al., 1993) and locomotory behaviors in the worm have been modeled previously (Niebur and Erdös, 1991, 1993; Erdös and Niebur, 1993).

The demonstration that the worm is an adaptive system (Rankin et al., 1990) is interesting because sophisticated nematode neurobiology and genetics allow the possibility of describing the nature of this plasticity at a circuit and cellular/molecular level. Many of the demonstrations of learning in this organism have been made through the study of the simple tap withdrawal reflex, for which the underlying neural circuitry is identified (Wicks and Rankin, 1995a). Nlthough the detail of the anatomical data used to describe this circuit is considerable, the functional polarities of the neurons of this circuit are unknown. This report describes an array of putative polarities for synapses of the tap withdrawal circuit. These predictions were made by optimizing the behavior of a modeled circuit under conditions of degradation isomorphic to laser ablation of the circuitry underlying the tap withdrawal behavior of real animals. Additionally, this model was used to investigate roles for two novel mechanosensory integration neurons.

\section{MATERIALS AND METHODS}

The model. 'The model used was a physiologically motivated one. However, in the absence of detailed physiological data from $C$. elegans, it was necessary to make a number of extrapolations from the related nematode Ascaris lumbricoides. These assumptions are presented in physiological rather than mathematical form to ensure that they are realistic. Further- 


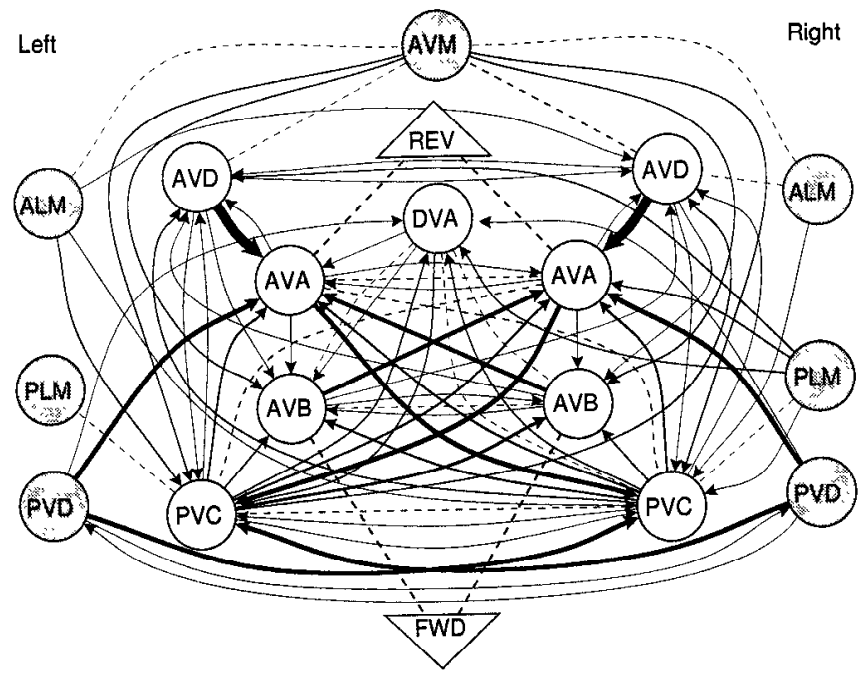

Figure 1. The complete connectivity of the tap withdrawal circuit. The circuit consists of seven sensory neurons (shaded circles), nine interneurons (unshaded circles), and two motorneuron pools (not shown), which produce forward and backward locomotion (triangles). Chemical connections are indicated by arrows, with the number of synaptic contacts being to the width of the arrow. Gap junctions are indicated by dotted lines. Every connection represented in this figurc was also rcpresentcd in the model. This representation is useful for identifying connection asymmetries, which might underlie the origin of oscillations that control locomotion and are hidden in simpler views of the circuitry.

more, preliminary investigations suggested that polarity predictions based on the modeled circuit were more strongly determined by circuit connectivity than the exact values of parameters used. Thus, approximate ranges for these parameters rather than precise values were derived. The effects of varying some of the more uncertain parameters were then assessed by rerunning the same experiments with values of these parameters varied over three orders of magnitude.

The circuitry was constructed by extracting connectivity data from AY's Neuroanatomy for Computation (Achacoso and Yamamoto, 1992). This data indicated not only the presence or absence of a set of synaptic contacts between a pair of neurons, referred to here as a synaptic class, but also incorporated the actual number of documented electrical and chemical connections within that synaptic class. Each synaptic contact within a class of synapses was assigned the same reversal potential and conductance as all other synapses within that class. This enabled the simple construction of complex circuits in which all documented synapses (including all bilateral asymmetries) were included in the model. It was assumed that the functional efficacy of a synaptic class was correlated with the number of contacts observed within that synaptic class. Thus, circuits constructed in this way possessed connections witl weights deternined by anatomical criteria. These weights were not varied further in this model; only the reversal potential, which determined the sign of the connection, was allowed to vary. 'The complete connectivity of the modeled tap withdrawal circuit is shown in Figure 1.

The model was based on all available physiological and anatomical data from $C$. elegans and the related nematode, Ascaris. The physiological parameters used in the derivation of the data presented in this report are shown in Tables 1 and 2. The model was implemented in Objective-C on Intel-486, HP series 9000 and NeXT computers running NEXTSTEP software, and was integrated using fourth-order Runge-Kutte (Press et al., 1989) to an accuracy of $0.5 \%$.

The neuron. The neurons of $C$. elegans have simple morphologies, which are preserved across individuals. Many neurons consist of a single unbranched process, and few have more than two branches (Chalfie and White, 1988). Electrophysiology on C. elegans cells is still in its infancy (however, see Raizen and Avery, 1994; Avery et al., 1995), and little is known about the membrane characteristics of its neurons. However, electrophysiology has been done on Ascaris, a larger nematode related to C. elegans (Davis and Stretton, 1989a,b). Its dorsal and ventral nerve cords have been reconstructed and show considerable similarity to those of $C$. elegans, and homologs of C. elegans motor neurons have been found
Table 1. Average simulation parameters

\begin{tabular}{lll} 
Neuron parameters & Value & Units \\
\hline Membrane resistance & See Table 2 & Ohms \\
Membrane capacitance & See Table 2 & Farads \\
Membrane leakage potential & -0.035 & Volts \\
Synaptic parameters & & \\
EPSP reversal potential & 0.00 & Volts \\
IPSP reversal potential & -0.048 & Volts \\
Synaptic conductance & $6.00 \mathrm{E}-10$ & Siemens \\
$V_{\text {Range }}$ & 0.035 & Volts \\
Gap junction conductance & $5.00 \mathrm{E}-09$ & Siemens \\
Tap parameters & & \\
Pulse rest & 0 & Amps \\
Phasic pulse & $1.00 \mathrm{E}-11$ & Amps \\
Start time & 0.01 & Sec \\
Duration & 0.3 & Sec \\
Tonic pulse & $2.50 \mathrm{E}-10$ & Amps
\end{tabular}

The list of physiological parameters used in the four experiments run in this report are summarized. For a more detailed discussion of the origin of these values, see Materials and Methods.

in Ascaris (Chalfie and White, 1988; Stretton et al., 1992). For this model, electrophysiological data from Ascaris was used to determine model parameters.

Evidence fron Ascaris suggests that signal propagation in C. elegans neurons is likely accomplished electrotonically, without classical all-ornone action potentials. Intracellular recordings of Ascaris motorneurons and interneurons show no evidence of action potentials, and it has not been possible to evoke them (Davis and Stretton, 1989a). Membrane resistivity in Ascaris is unusually high $\left(60-300 \mathrm{k} \Omega \mathrm{cm}^{2}\right)$ and is within the range that would permit signal propagation without action potentials. Niebur and Erdös (1993) have used Ascaris data to do detailed computational studies of the electrotonic characteristics of $C$. elegans neurons and have shown that the integration of $C$. elegans locomotion can be accounted for by purely electrotonic signals.

Davis and Stretton (1989a) have measured specific membrane resistances $\left(\hat{R}_{m}\right)$ and intracellular resistivity $\left(\hat{R}_{i}\right)$ in Ascaris. In four motorneurons, $\hat{R}_{m}$ varied from 89 to $251 \mathrm{k} \Omega \mathrm{cm}^{2}$, and $\hat{R}_{l}$ from 79 to $314 \Omega \mathrm{cm}$. We assumed that membrane properties in C. elegans were similar and used an average of the four measurements: $\hat{R}_{i}=180 \Omega \mathrm{cm}$ and $\hat{R}_{m}=150 \mathrm{k} \Omega \mathrm{cm}^{2}$. We assumed a specific membrane capacitance of $1 \mu \mathrm{F} / \mathrm{cm}^{2}$, a standard value for a lipid bilayer (Rall, 1989). These membrane properties were

Table 2. Summary of single-neuron characteristics

\begin{tabular}{lllllll} 
Cell & $\begin{array}{l}\text { Primary } \\
\text { process } \\
(\mathrm{mm})\end{array}$ & $\begin{array}{l}\text { Secondary } \\
\text { process } \\
(\mathrm{mm})\end{array}$ & $\begin{array}{l}\text { Surface } \\
\text { area } \\
\left(10^{-6} \mathrm{~cm}^{2}\right)\end{array}$ & $\begin{array}{l}C_{m} \\
(\mathrm{pF})\end{array}$ & $\begin{array}{l}R_{m} \\
(\mathrm{G} \Omega)\end{array}$ & $\frac{V(l)}{V_{n}}$ \\
\hline ALM & 0.50 & 0.03 & 9.1 & 9.1 & 16 & 0.89 \\
PLM & 0.48 & 0.06 & 9.1 & 9.1 & 16 & 0.9 \\
AVM & 0.24 & 0.03 & 5.0 & 5.0 & 30 & 0.97 \\
PVD & 0.74 & 0.22 & 16 & 16 & 9.4 & 0.78 \\
PVC & 0.96 & - & 16 & 16 & 9.4 & 0.68 \\
AVA & 0.93 & - & 15 & 15 & 10 & 0.69 \\
AVB & 0.86 & - & 14 & 14 & 11 & 0.73 \\
AVD & 0.86 & - & 14 & 14 & 11 & 0.73 \\
DVA & 0.91 & - & 15 & 15 & 10 & 0.70
\end{tabular}

Branching morphology and process length were taken from Wood (1988) and White et al. (1986), assuming a standard worm length of $1 \mathrm{~mm}$. An average process diameter of $0.5 \mu \mathrm{m}$ was obtained from measurements of electron micrographs in White et al. $(1976,1986)$. An average soma diameter of $5 \mu \mathrm{m}$ was measured from camera lucida drawings in Wood (1988). $V(l) / V_{\omega}$ is the attenuation of a voltage clamp $V_{o}$ along the full length of the primary process according to a sealed-end cable equation (Rall, 1989) and gives an indication of a cell's isopotentiality. 
adapted to $C$. elegans anatomy by using the surface area of each cell (see Table 2). Each neuron's branching morphology is given in Wood (1988) and White et al. (1986). This, together with measurements of electron micrngraphs in White et al. $(1976,1986)$, were used to determine average process lengths and diameters. Diameters varied from 0.2 to $1.0 \mu \mathrm{m}$, and an average value of $d=0.5 \mu \mathrm{m}$ was used.

Process lengths were taken from diagrams in Wood (1988), assuming a standard worm length of $1 \mathrm{~mm}$. Soma diameters were taken from camera lucida drawings in Wood (1988). Soma diameters varied from 2 to $10 \mu \mathrm{m}$; we used an average diameler of $5 \mu \mathrm{m}$. From these data, a tolal membrane surface area for each cell was computed, and the resulting total membrane capacitance and resistance for the entire cell was derived (see Table 2). For simplicity, it was assumed that cells were isopotential. Because the length constant (Rall, 1989)

$$
\lambda=\frac{1}{2} \sqrt{\frac{d \hat{R}_{m}}{\hat{R}_{\imath}}}
$$

was generally longer than the process (data not shown), this was reasonable (also see Table 2). Thus, a neuron's membrane potential, $V$, was governed by the usual single-compartment membrane equation (Segev et al., 1989):

$$
C_{m} \frac{d V}{d t}=\frac{1}{R_{m}}\left(V_{\mathrm{LEAK}}-V\right)+\sum I_{\mathrm{SYN}}+I_{\mathrm{EXT}},
$$

where $C_{m}$ is the total membrane capacitance for the cell, $R_{m}$ is the total membrane leakage resistance for the cell, $V_{\text {LEAK }}$ is the leakage potential of the cell, $I_{\mathrm{SYN}}$ is the current attributable to a synaptic input, and $I_{\mathrm{EXI}}$. is any injected current. A value of $-35 \mathrm{mV}$ was used for the leakage potential in these cells (R. E. Davis, personal communication).

Gup junctions. The andonical reconstruction of the nematode nervous system allowed the identification of both electrical and chemical synapses (White et al., 1986). Gap junctions were modeled as ohmic resistances, where current flowing into cell $i$ from cell $j$ was given by:

$$
\hat{I}_{\imath}=\hat{g}_{t !}\left(V_{1}-V_{i}\right),
$$

where $\hat{g}_{i j}$ was the conductance of the gap junction. Niebur (1988) used a standard conductance per unit area value of $1 \mathrm{~S} / \mathrm{cm}^{2}$ (Bennett, 1972) and used unpublished micrographs to determine the area of each gap junction. He reported that gap junctions ranged from 0.2 to $2 \mu \mathrm{m}$ long and were $0.5 \mu \mathrm{m}$ wide (Niebur, 1988). In our model, a standard gap junction length of $1 \mu \mathrm{m}$ was assumed, with a resulting conductance of $5 \mathrm{nS}$ for all gap junctions. In some experiments, this value was increased or decreased by a factor of 10 to test the sensitivity of the model's predictions to the precise value of the conductance used.

Synapses. Synaptic classes consisted of a number of individual synaptic contacts. The number of contacts in each class was extracted from an anatomical database of $C$. elegans synaptic connectivity (Achacoso and Yamamoto, 1992). The identification of chemical synapses from the anatomical reconstruction was done by identifying presynaptic specializations and inferring postsynaptic partners based on proximity; no postsynaptic specializations were evident in the electron microscope reconstructions (White et al., 1986). Any error associated with this technique would tend to overestimate the number of chemical synapses in the organism, however, and any spurious synaptic classes would not be duplicated with high frequency. Therefore, such synapses, although present in the model, would not be assigned a large synaptic weight and thus would not have a large impact on the response of the circuit to stimulation. Each modeled synapse represented a class of synaptic contacts with total synaptic conductance - the "weight"-given by the product of the number of individual contacts within the class and the individual synaptic conductance.

The synapse model used was based on the graded synapse model used by Lockery and Sejnowski (1992) in the leech local bending circuit. However, it was extended to explicitly include the synaptic reversal potential as well as the conductance. Postsynaptic current was attributable to gated channels in the postsynaptic membrane with inward current given by:

$$
I=g(t)\left(E_{\mathrm{SYN}}-V_{\mathrm{POST}}\right),
$$

where $g(t)$ is the synaptic conductance of the postsynaptic membrane, which was gated according to presynaptic potential, and $E_{\mathrm{SYN}}$ is the reversal potential for the synaptic conductance, which was assumed to be constant. For excitatory synapses, a reversal potential of $0 \mathrm{mV}$ was used, and for inhibitory synapses $-45 \mathrm{mV}$ was used (R. E. Davis, personal communication). For simplicity, it was assumed that all synapses made by a single cell possessed the same reversal potential. This amounts tn assuming that all synapses made by a given presynaptic cell were of the same polarity and class. Computationally, this reduced the number of optimized parameters each of which possessed two possible values to the number of neurons in the modeled circuit. It was further assumed that all modeled synapses functioned as fast ligand-gated channels. It was possible that some andumically defined synapses were modulatory and acted via slow second-messenger systems, or that synaptic function was altered by the milieu interieur (Harris-Warrick et al., 1992); however, we assumed that these modulatory effects did not affect a single tap withdrawal response.

In the leech local bending reflex, Lockery and Sejnowski (1992) observed multiple time courses in some of the postsynaptic responses, and to model this they used a slow $(10 \mathrm{msec})$ and a fast $(1500 \mathrm{msec})$ conductance, each governed by its own first-order equation. Preliminary versions of this model used a fast $(10 \mathrm{msec})$ synaptic time constant, but no significant differences were noted in the results of circuits containing these synapses and simulations, which used an instantaneous synapse. We therefore used a synaptic conductance that depended only on the presynaptic membrane potential:

$$
g(t)=g_{\infty}\left(V_{\mathrm{PRE}}\right),
$$

where $g_{\infty}$ represents the steady-state conductance in response to a presynaptic voltage.

Synaptic activation. No direct recordings have yet been made in $C$. elegans to determine properties of synaptic activation. In recordings made from Ascaris commissural motorneurons, Davis and Stretton (1989b) demonstrated that synaptic transmission is graded and transmitter is released tonically between both excitatory and inhibitory motorneurons and postsynaptic muscle and motorneurons. They found that changes in postsynaptic potential were related to presynaptic depolarizing current by a sigmoidally shaped curve and that the presynaptic resting potential lies approximately in the middle of the voltage-sensitive range of synaptic transmission.

Dynamic network simulations based on graded synaptic transmission have been described previously (Lockery and Sejnowski, 1992; DeSchutter et al., 1993). We assumed that synaptic activation and transmission in C. elegans was similar to Ascaris; namely, that it was graded and sigmoidally shaped with presynaptic potential and was tonically active with the equilibrium potential in the middle of the voltage-sensitive range. Accordingly, we used a symmetric sigmoidal function to model the steadystate postsynaptic membrane conductance:

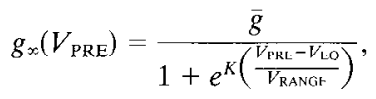

where $\bar{g}$ is maximal postsynaptic membrane conductance for the synapse, $V_{E O}$ is the presynaptic equilibrium potential at the middle of the voltagesensitive range, and $V_{\text {RANGE }}$ is the presynaptic voltage range over which the synapse activated. We used a value of

$$
K=2 \ln \left(\frac{0.1}{0.9}\right)=-1.3944
$$

so that the conductance changed from 10 to $90 \%$ of its maximal value over a presynaptic voltage range of $V_{\mathrm{RANGE}}$. Note that because synapses were tonically active, a cell's equilibrium potential was not defined solely by its resting membrane potential, but rather was determined from the fixed point of the entire system of equations governing the circuit. This was computed before each run in the following way.

Equilibrium potential. The assumption that tonically active synapses were active in the middle of their voltage-sensitive range at the equilibrium potential implied that the postsynaptic conductance $g(t)$ was onehalf its maximal value $\bar{g}$ when the circuit was at equilibrium. Let $\boldsymbol{V}_{i}^{r}$ denote the membrane potential for neuron $i$ and similarly for other quantities pertaining to neuron $i$. Let $I_{l y}$ denote the synaptic current flowing into neuron $i$ from neuron $j$ across a single synapse and let $\omega_{i j}$ denote the total number of synaptic connections from neuron $j$ to neuron $i$. Similarly, let $\hat{I}_{i j}$ denote current flow across a single gap junction in the direction from neuron $j$ to neuron $i$ and let $\hat{\omega}_{i j}$ denote the total number of gap junctions between neuron $j$ and neuron $i$. Finally, let $I_{i}$ denote the external current 
flow into cell $i$ (caused by either sensory stimulation or external current injection). Then, the entire system is given by:

$$
\begin{aligned}
& R_{m} C_{m_{1}} \frac{d V_{l}}{d t}=V_{\mathrm{LEAK},}-V_{i}+R_{m_{i}} \sum_{j=1}^{N}\left(I_{l j}+\hat{I}_{\imath j}\right)+I_{l}, \\
& \hat{I}_{l y}=\hat{\omega}_{i j} \hat{g}_{i j}\left(V_{j}-V_{i}\right), \\
& I_{l j}=\omega_{l j} g_{l j}\left(E_{l j}-V_{l}\right), \\
& \frac{d g_{i j}}{d t}=\frac{g_{x_{i j}}\left(V_{j}\right)-g_{i j}}{\tau_{i j}},
\end{aligned}
$$

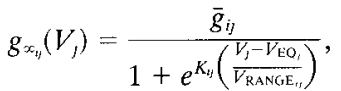

where $N$ is the number of neurons in the circuit and $\tau_{i j}$ is the synaptic time constant.

At equilibrium, $V_{t}=V_{\mathrm{EQ}_{i}}$, and $d V / d t, d g_{i j} / d t$ and $I_{i}$ are zero. Synaptic conductances are tonic and are at their half-activation at equilibrium so that

$$
g_{x_{y}}\left(V_{\mathrm{EQ}_{3}}\right)=\frac{\bar{g}_{i I}}{2} .
$$

After algebraic manipulation, this yields a system of linear equations that can be solved using standard Gaussian elimination to find $V_{\mathrm{EQ}}$ (Press et al., 1988):

$$
\mathbf{V}_{\mathrm{EQ}}=\mathbf{A}^{-1} \mathbf{b},
$$

where $A_{i j}$ is the $i$ th row and $j$ th column of matrix $\mathbf{A}$ and is given by:

$$
\begin{gathered}
A_{i j}=-R_{m_{i}} \hat{\omega}_{i j} \hat{g}_{l j}, \quad i \neq j, \\
A_{i t}=1+R_{m_{i}} \sum_{j=1}^{N}\left(\hat{\omega}_{l j} \hat{g}_{i j}+\omega_{i l} \tilde{g}_{i j} / 2\right)
\end{gathered}
$$

and $\mathbf{b}$ is a vector that is given by:

$$
b_{\iota}=V_{\text {LEAK }_{t}}+R_{m_{t}} \sum_{j=1}^{N} E_{i j} \omega_{i j} \bar{g}_{i j} / 2 .
$$

The computed equilibrium potential of cells varied within a physiologically realistic range from -47 to $0 \mathrm{mV}$, with a mean of $-24 \mathrm{mV}$ and an $\mathrm{SD}$ of $13 \mathrm{mV}$. This did not vary appreciably from cell to cell, but rather depended on the circuit's polarity configuration and ablation condition. As an aside, the system (Eq. 14) can also be inverted to explicitly give $V_{\mathrm{LEAK}}$ in terms of $V_{\mathrm{EQ}}$ :

$$
\begin{aligned}
V_{\mathrm{LEAK}_{t}}=V_{\mathrm{EQ}}-R_{m_{t}} \sum_{j=1}^{N} \omega_{i j} \bar{g}_{l j} / 2\left(E_{l j}\right. & \left.-V_{\mathrm{EQ}}\right) \\
& -R_{m_{i}} \sum_{j=1}^{N} \hat{\omega}_{l} \hat{g}_{g^{\prime}}\left(V_{\mathrm{EQ}}-V_{\mathrm{EQ}}\right) .
\end{aligned}
$$

Synaptic parameters. To determine values for $V_{\mathrm{RANGF}}$ and $\bar{g}$, the synapse model was fitted to published measurements (Davis and Stretton, 1989b) of Ascaris muscle cell postsynaptic response to presynaptic current injection as detailed below. Thus, values for $V_{\text {RANGE }}$ and $\bar{g}$ for particular Ascaris synapses were obtained. We assumed that $C$. elegans synapses activated over voltage ranges similar to Ascaris synapses. However, the maximal synaptic conductance, $\bar{g}$, nceded to be adapted to C. elegans. We assumed that $\bar{g}$ represented the product of a synaptic conductance per unit area and a synaptic area. In the case of a synapse mediated by a single population of ion channels, $\bar{g}$ would be equivalent to the singlechannel conductance of an open channel multiplied by the total number of channels. To adapt the $\vec{g}$ value from Ascaris to C. elegans, we assumed that $C$. elegans synapses had similar unit-area conductances and accordingly scaled the Ascaris $\bar{g}$ by a factor to account for the presumed difference in synaptic areas. We assumed that the total synaptic area between two cells was proportional to the length of the process, which we estimated by the ratio of body lengths-approximately $1 / 250$. As this represents only a gross approximation, the value of $\bar{g}$ used in these studies was varied over three orders of magnitude in different experiments (see Results)

Modeling Ascaris monosynaptic responses. Data from Davis and Stretton (1989b; their Figs. 13 and 14) show the postsynaptic response of an Ascaris dorsal muscle cell (DM) to current injected into a presynaptic excitatory motorneuron, DE1, and the response of a ventral muscle cell (VM) to current injected into a presynaptic inhibitory motorneuron, VI. Both of these response profiles were sigmoidal in shape, were centered approximately at the resting potential, and had asymptotic saturated postsynaptic responses at the extremes of positive and negative presynaptic current injection. Therefore, the sigmoidal tonic response model presented here was well suited to fitting these synaptic responses.

Davis and Stretton $(1989 \mathrm{a}, \mathrm{b})$ placed a recording electrode in a muscle cell within the output zone of the motorneuron, and an injecting electrode at the ventral end of a commissural process leading to the synapse. The measured input resistance of the motorneuron was used to obtain the resulting membrane potential at the point of current injection, and an infinite cable model was used to determine the membrane potential at the presynaptic site. Because the recordings that were used to determine input resistance were made at the same ventral end of the commissure as was injected for the synaptic response measurements (R. E. Davis, personal communication), and because the input resistance was approximately constant over the relevant range of injected current (Davis and Stretton, 1989a), it is possible to directly use the measured input resistance to determine the membrane potential at the point of current injection.

Davis and Stretton (1989a) determined the motorneuron cable properties by fitting their measurements along the commissure to an infinite cable model (Rall, 1989) and found that the length constant was unusually high $(\lambda \sim 8 \mathrm{~mm})$-approximately the same magnitude as the length of the process. With such a large length constant, it is possible that the cable's branching morphology and sealed ends played a significant role in determining these cable properties (Rall, 1977), suggesting that a sealedcnd cable model might be more appropriate. However, for consistency we used an infinite cable model with cable constants as determined by Davis and Stretton (1989a) to reproduce their measured voltage response along the commissure.

Specifically, the presynaptic depolarization in response to an injected current is given by:

$$
\Delta V_{\mathrm{PRE}}=I_{\mathrm{INJ}} R_{\mathrm{PRE}} e^{-L / \lambda},
$$

where $L$ is the distance from the point of current injection to the synapse and $R_{\mathrm{PRE}}$ is the input resistance at the point of injection. For the DE1-DM synapse, the distance, $L$, was 5-8 $\mathrm{mm}$; for the VI-VM synapse, it was $0.5-2.5 \mathrm{~mm}$ (R. E. Davis, personal communication). We used the mean of 7 and $1 \mathrm{~mm}$, respectively. The input resistances for the DE1 and VI motorneurons were reported to be 6 and $17 \mathrm{M} \Omega$, respectively (Davis and Stretton, 1989a).

According to the sigmoidal tonic response model, the steady-state plateau response of the postsynaptic muscle is given by:

$$
V_{\mathrm{POST}}=V_{\mathrm{LEAK}}+R_{\mathrm{POST}} n g_{\infty}\left(\Delta V_{\mathrm{PRE}}\right)\left(E_{\mathrm{SYN}}-V_{\mathrm{POST}}\right),
$$

where $V_{\mathrm{LEAK}}$ and $E_{\mathrm{SYN}}$ pertain to the postsynaptic muscle cell, $R_{\mathrm{POST}}$ is the postsynaptic cell's input resistance, $g_{\infty}\left(V_{\mathrm{PRE}}\right)$ is the steady-state synaptic conductance, and $n$ is the total number of synapses between the motorneuron and the muscle cell. The input resistance of VM and DM was measured to be $0.18-0.50 \mathrm{M} \Omega$, with a mean of $0.3 \mathrm{M} \Omega$ (R. E. Davis, pcrsonal communication). Values of $V_{\mathrm{LEAK}}=-35 \mathrm{mV}$ and $E_{\mathrm{SYN}}-0 \mathrm{mV}$ for excitatory and $E_{\mathrm{SYN}}=-45 \mathrm{mV}$ for inhibitory reversal potentials were used (R. E. Davis, personal communication). A light microscope study of dye-injected muscle cells in Ascaris suggested that the DE1 motorneurons make approximately 5-10 synapses to each DM and the VI motorneurons make approximately 8-16 synapses to each VM (J. Donmoyer, personal communication). Values from the high end of these ranges were adopted for $n$ in Equation 20 because muscle cells form gap junctions with one another (J. Donmoyer, personal communication), and thus it was possible that synapses from neighboring muscle cells played a role in determining a muscle cell's postsynaptic potential.

Equation 20 can be rearranged to yield values of $V_{\text {POST }}$ explicitly in terms of $V_{\mathrm{PRE}}$ :

$$
V_{\mathrm{POST}}=\frac{V_{\mathrm{LEAK}}+E_{\mathrm{SYN}} R_{\mathrm{POST}} n g_{x}\left(\Delta V_{\mathrm{PRE}}\right)}{1+R_{\mathrm{POST}} n g\left(\Delta V_{\mathrm{PRE}}\right)},
$$


where

$$
g_{\approx}\left(\Delta V_{\mathrm{PRE}}\right)=\frac{\bar{g}}{1+e^{K_{V_{\mathrm{RANGI}}}^{\Delta V_{\mathrm{PRL}}}}}
$$

The change in postsynaptic potential was therefore given by:

$$
\Delta V_{\mathrm{POST}}=V_{\mathrm{POST}}-V_{\mathrm{EQ}},
$$

where $V_{\mathrm{EQ}}$ is the equilibrium potential achieved by the postsynaptic cell under unstimulated in-circuit tonic synaptic input (see Eqs. 14-18) and is given by:

$$
V_{\mathrm{EQ}}=\frac{V_{\mathrm{LEAK}}+E_{\mathrm{SYN}} R_{\mathrm{POST}} n \bar{g} / 2}{1+R_{\mathrm{POST}} n \bar{g} / 2}
$$

Equations 19 and 21-24 define a nonlinear function for postsynaptic membrane potential in terms of presynaptic injected current and contain two unknown parameters: $\bar{g}$ and $V_{\text {RANGE. Levenberg-Marquardt's }}$ method (Press et al., 1988) was used to fit this function to the data from Davis and Stretton (1989b).

We obtained good results to the fit for the inhibitory VI-VM synapse. This fit included the reversal potential $E_{S Y N}$ as a fit parameter; this improved the fit substantially without significantly changing the reversal potential $(-48 \mathrm{mV}$ as opposed to $-45 \mathrm{mV})$. The results of this fitting procedure yielded values of $\bar{g}=150 \mathrm{nS}, V_{\mathrm{RANGE}}=52 \mathrm{mV}, E_{\mathrm{SYN}}=-48$ $\mathrm{mV}$ and were stable under various initial conditions. The DE1-DM fit was less precise because the steady-state conductance was not precisely a symmetric sigmoid. We therefore manually explored a number of parameter values and attempted to reproduce the approximate range and amplitude of activation. For this we obtained values of $V_{\text {RANGE }}=20 \mathrm{mV}$ and $\bar{g}=90 \mathrm{nS}$.

To adapt these values to $C$. elegans, an average of the two $V_{\text {RANGE }}$ values was used to estimate the activation range $(-35 \mathrm{mV})$, and the $\bar{g}$ from the VI-VM synapse fit was scaled by the $1 / 250$ ratio of body lengths to yield a maximal conductance of $0.6 \mathrm{nS}$ for an individual $C$. elegans synapse.

The gearbox. This model does not explicitly incorporate nematode locomotion; these issues have been dealt with adequately elsewhere (Niebur and Erdös, 1991, 1993; Erdös and Niebur, 1993). Rather, this report concentrates specifically on sensorimotor integration. However, because a behavioral variable was used to optimize the modeled output, it was necessary to rigorously define the relationship between the animal's locomotion and activation of the circuitry that controls that behavior. This issue was addressed with simple assumptions, which were consistent with work on the modeling of nematode locomotion (Niebur and Erdös, 1991, 1993; Stretton et al., 1992; Erdös and Niebur, 1993) and current theories of tap withdrawal circuit function (Chalfie et al., 1985; Wicks and Rankin, 1995a). The outpul of the tap withdrawal circuit was assumed to control locomotory behavior primarily through the action of the interneurons AVB and AVA. These two interneurons make electrical connections with motor neurons all along the ventral cord of the worm. The AVA interneurons make gap junctions with the motor neurons AS, VA, and DA, which are presumed to excite backward locomotion; the AVB interneurons form gap junctions with the motor neurons VB and DB, which are presumed to excite forward locomotion. Ablation of these cells almost completely destroys an animal's ability to move forward (in the case of AVB ablations) or backward (in the case of AVA ablations) (Chalfie et al., 1985; Wicks and Rankin, 1995a). Thus, it was simply assumed that the degree to which an animal reversed was proportional to the depolarization of the AVA interneuron and inversely proportional to the depolarization of the AVB interneuron. Forward locomotion in response to tap was also proportional to this value; a lower propensity to reverse was equivalent to a higher propensity to accelerate. The exact nature of this proportionality was not defined because in vivo it will be modulated by a number of neural, hydrostatic, and physical forces that are beyond the scope of this endeavor. The gearbox, i.e., the transformation equation that was used to convert depolarization of AVA and AVB into behavior, was simply:

$$
\text { Propensity to Reverse } \propto \int V_{\mathrm{AVB}}-V_{\mathrm{AVA}} d t \text {. }
$$

The integration was calculated from the time of the tap stimulation until either the end of the simulation or until the integrand changed sign.
Additionally, the test for a change of integrand sign was suppressed for a grace period of $100 \mathrm{msec}$ to allow for initial transients after the tap.

One consequence of the gearbox assumption is that, because of uncertainty regarding the exact nature of the proportionality between the output of the AVA and AVB interneurons and the magnitude of the evoked behavior, comparisons of model data and empirical data must be limitcd to relative changes in rcsponsc magnitudc. Thus, such comparisons were made between data profiles that had been normalized about the mean of that polarity configuration's response level (see below). This measure detected changes in the levels of responding to tap produced by an ablation series, without being sensitive to the absolute response magnitude of a particular circuit configuration-information which in any case is meaningless in the context of the gearbox assumption.

Strategy for neuron polarity determination. The animal's response to a light mechanosensory stimulus has been intensively studied (Chalfie and Sulston, 1981; Chalfie et al., 1985; Wicks and Rankin, 1995a). Specifically, Chalfie and colleagues have described the circuitry that underlies the worm's reflexive response to a light touch to either its head or tail. This touch circuit was the starting point for the delineation of the circuitry responsible for the animal's response to a mechanically delivered stimulus (i.e., "tap") to the side of the substrate on which the animal moves. An intact worm will generally respond to a tap stimulus with a cessation of forward motion, a reversal through some distance, and a resumption of forward locomotion in a new direction. This response has been termed the tap withdrawal reflex (Rankin et al., 1990). The magnitude of the tap withdrawal reflex can be quantified by measuring the distance through which an animal reverses.

The circuitry underlying the tap withdrawal reflex (see Fig. 1) (Wicks and Rankin, 1995a) has been identified using the laser ablation technique (Sulston and White, 1980; Avery and Horvitz, 1987, 1989). Ablations of neurons in this circuit can quantitatively and qualitatively alter an animal's response to tap. Three neuron classes, each composed of one or two cells, transduce the tap stimulus and segment the animal into two mechanosensory fields; anterior mechanosensory stimuli are transduced by the ALM and AVM cell classes, and the posterior input is transduced by the PLM cell class. The AVA and AVB interneurons are required for normal locomotion, and the AVD and PVC interneurons couple anterior and posterior mechanosensory input, respectively, onto AVA and AVB.

The ablation strategy previously used to describe the tap withdrawal circuit resulted in the accumulation of data sets that represented the worm's response to a tap stimulus when individual neurons in the tap withdrawal circuit were destroyed with a laser mircobeam (see Fig. 2) (Wicks and Rankin, 1995a). For example, the ablation of either the ALM sensory neuron class alone or in conjunction with the AVM sensory neuron resulted in animals that accelerated forward, rather than reversed, in response to a tap. Furthermore, the magnitude of both the reversal and acceleration behaviors elicited by a tap stimulus was modulated by ablation of the tap withdrawal circuit neurons. For example, the reversal response of animals lacking the PLM sensory neuron was larger than the reversal response of control animals with an intact circuit. This difference was presumed to be attributable to the loss of excitatory electrical input from the PLM sensory neuron to the PVC interneuron, as well as chemical input to the AVA and AVD interneurons (see Fig. 1). This chemical input could be either excitatory or inhibitory, and that variable should, in theory, affect the magnitude of the reversal response observed. Similarly, the ablation of both the ALM and AVM sensory neurons resulted in larger accelerations in response to a tap than did the ablation of ALM alone.

The connectivity of the worm's nervous system has been well described. However, the signs or polarities of the synapses, excitatory or inhibitory, which determine the behavior produced by a given circuit, are unknown. This set of synaptic signs is referred to in this report as the polarity configuration of that circuit. The same connectivity may result in many distinct behavioral outcomes depending on the particular polarity configuration that the circuit possesses. The data that represent the animal's tap withdrawal behavior after neuronal ablation-presented in Figure 2 -reflect the polarity configuration of the worm's tap withdrawal circuit; it is a representation of low the circuit that is defined by that set of polarities responds, in various states of degradation, to a tap stimulus. Another circuit-one with identical connectivity but a different polarity contiguration-would not necessarily yield the same profile of tap responses as a result of that series of lesions. Indeed, each possible polarity configuration of the tap withdrawal circuit might result in a distinctive pattern of behavior in response to the ablation of individual, or small sets of, neurons. This profile of behavior (see Fig. 2), representing the real 


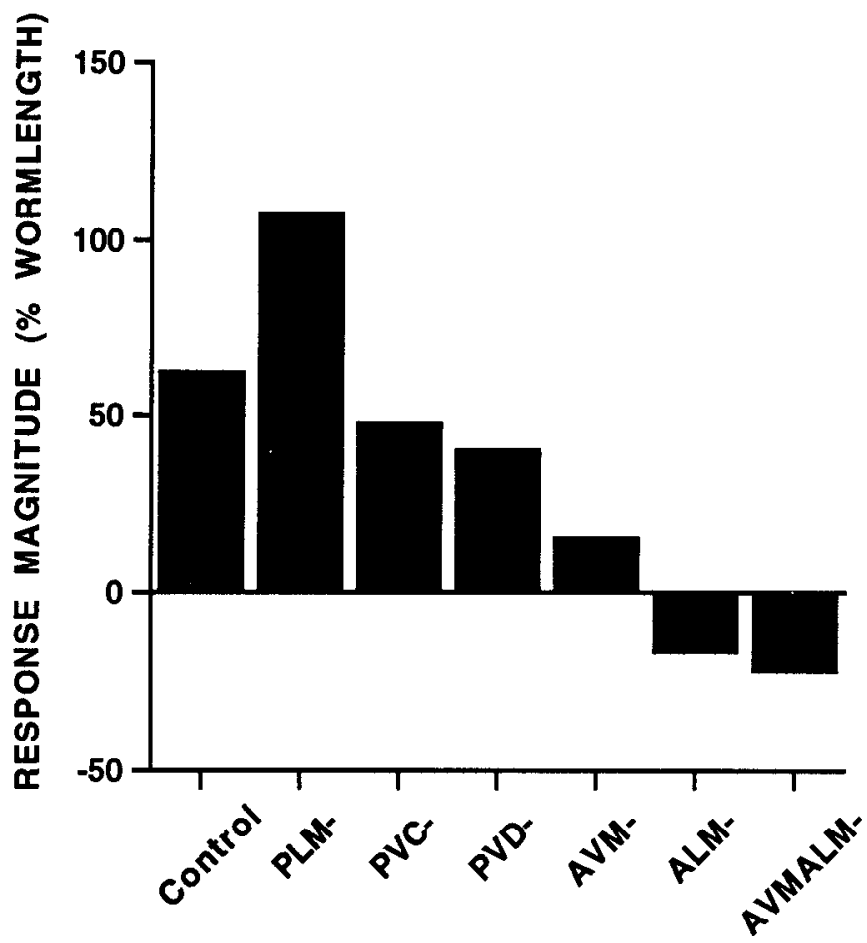

Figure 2. Mean response magnitudes of the tap withdrawal reflex of seven groups of animals. These data were used to optimize the array of underlying functional polarities of the modeled circuit. Some ablations (for example, the removal of PLM) resulted in larger reversal responses than in control animals; other ablations resulted in consistent accelerations in response to tap (indicated by a negative reversal magnitude for the ALM and AVMALM groups). Note that the acceleration measure is a change in velocity, whereas the reversal measure is a distance; these are not directly comparable. Thus, the ordinate represents a number that is proportional to the amount of forward or backward locomotion. It was assumed that the pattern of response represented on this figure was dictated partially by the array of synaptic signs, which constituted the underlying circuitry (see Materials and Methods, Strategy for neuron polarity determination). These data are adapted from Wicks and Rankin (1995a).

animal's response to a tap in a variety of ablation conditions, was used to find the best match within the space of all possible polarity configurations using a computational model of the tap withdrawal circuit.

We constructed a model of the tap withdrawal circuit and produced lesions in that circuit that were isomorphic to the ablation conditions represented in Figure 2. We then determined the profile of behavior for all seven ablation conditions predicted by the model for an arbitrary polarity configuration. The fitness of that polarity configuration was determined by an error function with three terms (see Eq. 26), each of which used a least-squares error approach to compare the model data profile for that configuration and the ablation data profile (see Fig. 3). Then, all possible polarity configurations were exhaustively enumerated and sorted according to their fitness. The response profiles (modeled and empirical) were compared by first separating those conditions that were associated with reversal responses from those that were associated with accelerations. Then these two profiles were standardized by expressing each data set as a series of Z-scores around the mean of each set of responses. This was done because we were interested in the relative change in the withdrawal response magnitude as a consequence of ablation, rather than the absolute value of the tap withdrawal response magnitude.

The first error term in the fitness function reflected how closely the ablation-induced modulation of modeled gearbox output matched the corresponding modulation of reversal behavior in real animals. 'The reversal profile comprised those ablation conditions that resulted in reversals in the real animal: the intact, PLM-, PVC-, PVD-, and AVM - conditions. Figure 3 shows three data sets that have been standardized to allow comparison. The empirical and modeled reversal pro- files were each standardized as described above and compared. The reversal error term was simply the sum of the least-squared errors between the two reversal profiles. The second error term in the fitness function reflected how well the modulation of the acceleration behavio of the model produced by ablation matched the corresponding modulation of acceleration behavior in real animals. The acceleration profile thus comprised the two ablation conditions, which resulted in animals that consistently accelerated in response to tap: the ALM - and AVMALM - ablations. Again, both the empirical acceleration profile (see Fig. 3) and the modeled acceleration profile were standardized as described above, and the least-squared error between the two acceleration profiles was derived for each configuration.

It was necessary to separate these two sets of comparisons because the acceleration and reversal behaviors were quantified with distinct measures (Wicks and Rankin, 1995) - an acceleration is a change in velocity, whereas a reversal is a distance. Although these two responses were not quantitatively comparable, it was still possible to qualitatively evaluate the AIM - and AVMALM - ablation conditions with respect to the reversal profile in the model. Any output from the gearbox (see Eq. 25) associated with the acceleration profile should, to be considered in accord with data from the real animal, be on average lower than the output of the gearbox associated with the reversal profile. It is not possible to make any statement with respect to how much smaller this gearbox output should be because of the incommensurability of the acceleration and reversal measures, but accelerations should be associated with relatively lower gearbox outputs than reversals. Thus, a third error term was derived to evaluate whether the response type produced by the model was in accord with the empirical data inasmuch as the gearbox output for these conditions was on average lower than for those ablation conditions associated with the reversal profile. The mean gearbox value for the reversal conditions (control, PLM -, PVD -, PVC-, and AVM-) was calculated for both the empirical data set in Figure 2 and the model data set. A similar mean acceleration value was calculated for the acceleration conditions in the two data sets. These two sets of values were converted to Z-scores, and a least-squares error between the model and empirical response type profile was calculated. Because there were only two terms in each of these distributions, this comparison was strictly qualitative. If the gearbox output for the acceleration conditions was, on average, lower than the gearbox output for the reversal conditions, the two normalized distributions would be identical, and the error contributed by this term would be zero. On the other hand, if the two acceleration conditions resulted in gearbox output that was, on average, larger than that produced by the reversal conditions, the error contributed by this term would be positive and furthermore, would be insensitive to how much larger the output was. Examples of two different response profiles from a typical experimentone representing a good fit and one representing a poor fit - are shown in Figure 3, along with the empirical data to which they were compared.

These three error terms were summed for each configuration. Thus, the fitness measure that was used to sort the list of polarity configurations was given by:

Filness $=E_{\text {Rcves sal Magnitude }}+E_{\text {Accclcration Magnitude }}+E_{\text {Response Type }}$,

where each term represented the least-squared error associated with the comparison of the model and empirical profiles for that behavioral measure. After summing the three error terms associated with each polarity configuration, the entire list of polarity configurations was sorted according to the resultant fitness function. The top 50 sorted polarity configurations from a single experiment are shown in Figure 4. The interpretation of this list was complicated by the fact that it was both exhaustive and complete. The top $n$ predictions, although sorted according to absolute fitness, may not have been significantly different from one another statistically. To address this issue, the polarity of each cell was analyzed independently. A given neuron was characterized as excitatory or inhibitory if it was determined that a given polarity for that cell was clustered at or near the top of the list. For example, a cell that was predicted to be inhibitory for the top 50 configurations was more likely to actually be inhibitory than a cell whose predicted polarity alternated through the top 50 configurations, even if in the case of the best configuration, it was also inhibitory.

A sign test (Siegel, 1956) is a nonparametric statistic that can be used to assess the probability that the two possible polarities $(+1$ or -1 in this case) appear with equal frequency within a tested fraction of this sorted list. A significant sign test indicates that one or the other sign clusters within that fraction of the sorted list at above chance levels. Thus, several one-sample sign tests were used for each neuron to determine whether a 


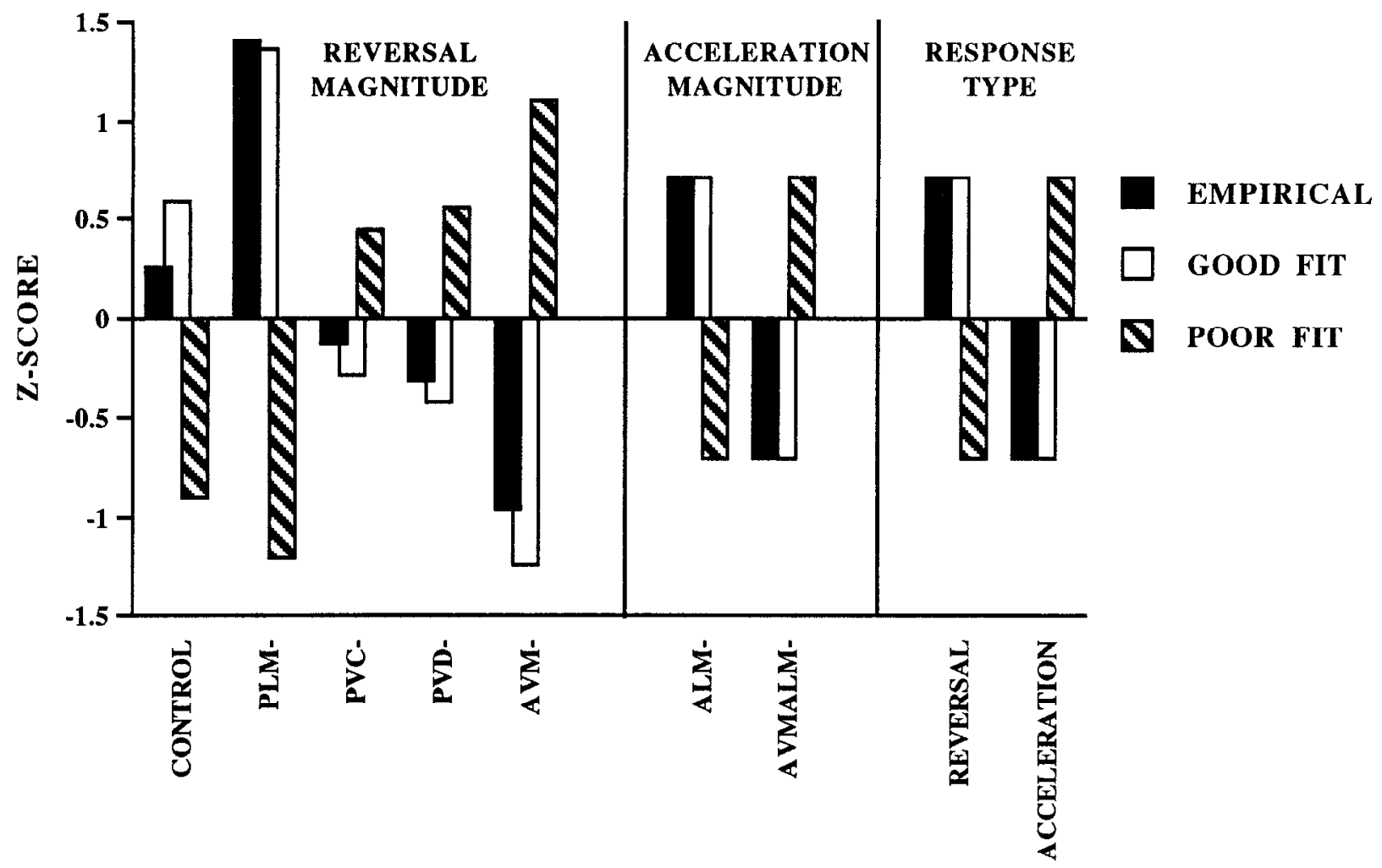

Figure 3. Two different response profiles from experiment 1 -one representing a good fit and one representing a poor fit-along with the empirical data to which they were compared. 'These data sets are expressed as three sets of standardized $L$-scores used to evaluate the relative modulation by ablation of three behavioral measures: REVERSAL MAGNITUDE, ACCELERATION MAGNITUDE, and RESPONSE TYPE. The first set of Z-scores incorporates those ablation configurations that result in a reversal response in the empirical data set shown in Figure 2 . It was used to determine the error associated with the relative modulation of reversal magnitude as a result of ablation between that data set and each polarity configuration from the model. The second set of Z-scores similarly assessed the error associated with the relative modulation of acceleration magnitude as a result of ablation. A third set of 7-scores evaluated the qualitative fit between the model and the empirical profiles with respect to response type. It assessed whether the gearbox output (Eq. 25) was lower, on average, for the acceleration profile than for the reversal profile, as was assumed to be the case for the intact animal. The fitness of a given configuration was calculated by summing the least-squared error between the model and empirical profiles for each of these three sets of standardized data. It is clear that the fitness of the modeled circuit to the behavioral data can be strongly modified by altering the array of underlying polarities in the modeled circuit.

given polarity for that cell clustered at a higher-than-chance frequency near the top of the sorted list of polarity configurations. This analysis was repeated for each of several fractions of the sorted list of configurations. Because the sorted list of configurations was complete, i.e., each configuration differed from all others, and because a significant prediction at one fraction of the list was indicative of a trend that might also be detected at other nearby fractions of the list if they were analyzed, this constitutes a conservative analysis of the data.

This paper reports the results of analyses based on four fractions of the list of sorted polarity configurations. Three of these (the top $10 \%$, first quartile, and top half) are arbitrary, but informative, and the fourth (designated " $\alpha$ ") was based on the shape of the frequency distribution of the fitness function that was used to sort the list of polarity configurations. The $\alpha$ fraction was defined as that fraction of the list of polarity configurations whose members possessed a fitness value that was $>1$ SD above the mean of the fitness frequency distribution. Thus, the fraction of the list that was defined hy $\alpha$ was dependent on the distribution of the error term used to sort the list. These fitness distributions are shown in Figure 5.

Modeling the tap stimulus. The tap stimulus was modeled as a phasic depolarization of the sensory neurons PLM, ALM, and AVM, which have been shown to mediate the response to tap in the intact animal (Wicks and Rankin, 1995a). However, it is likely that the tap does not represent the only input to the circuit in the intact animal. For example, the neuron PVD has been thought of as a stretch receptor (E. Hedgecock, cited in Way and (haltie, 1988) or a background mechanosensory input detector (Wicks and Rankin, 1995a). In addition, the interneuron DVA receives considerable synaptic input from chemosensory circuitry, and therefore may be a cell that modulates the activity of the tap withdrawal circuit according to the chemical milieu (Wicks and Rankin, 1995a). Therefore, four complete experiments were run to determine to what extent variations in the way these two cells were represented altered the nature of the polarity predictions. The effect of the inclusion of DVA was assessed by comparing two experiments in which DVA was included (experiments 2 and 4) with two experiments in which DVA was absent (experiments 1 and 3). Within each of these two conditions, the effect of the nature of the stimulation that PVD and DVA (when present) received was assessed. Two stimulation parameter sets were used. In the tonic condition (experiments 3 and 4), the PVD and DVA neurons were activated by a low (one-quarter pulse input magnitude) tonic stimulation that was continuously present and not correlated with the phasic tap input to the other touch neurons. These parameters were chosen to mimic the effects of an unspecified mechanosensory and chemosensory input to PVD and DVA, respectively. In the phasic condition, these two neuron classes were not explicitly stimulated (experiments 1 and 2).

Preliminary versions of these results have been reported previously in abstract form (Roehrig et al., 1994).

\section{RESULTS}

The results of the four experiments are summarized in Table $3 \mathrm{~A}-\mathrm{D}$. The table represents a series of probability values for sign tests conducted on the indicated percentages of the sorted lists of polarity configurations in each of four experiments: (A) the exclusion of DVA with no stimulation of PVD; (B) the inclusion of DVA in the circuit and no stimulation of DVA and PVD; (C) the exclusion of DVA with tonic activation of PVD; and (D) the inclusion of DVA and tonic stimulation of DVA and PVD. Taken together, the results of the four experiments made significant 


\begin{tabular}{|c|c|c|c|c|c|c|c|c|c|c|c|c|c|c|c|c|c|c|c|}
\hline & $\begin{array}{l}\text { A } \\
\text { L } \\
\text { M }\end{array}$ & $\begin{array}{l}\mathrm{P} \\
\mathrm{L} \\
\mathrm{M}\end{array}$ & $\begin{array}{l}\text { P } \\
\text { V } \\
\text { D }\end{array}$ & $\begin{array}{l}\text { A } \\
\text { V } \\
\text { B }\end{array}$ & $\begin{array}{l}\mathrm{P} \\
\mathrm{V} \\
\mathrm{C}\end{array}$ & $\begin{array}{l}\text { A } \\
\text { V } \\
\text { A }\end{array}$ & $\begin{array}{l}\text { A } \\
\text { V } \\
\text { D }\end{array}$ & $\begin{array}{l}\text { A } \\
\text { V } \\
\text { M }\end{array}$ & $\begin{array}{l}\text { D } \\
\text { V } \\
\text { A }\end{array}$ & & $\begin{array}{l}\text { A } \\
\text { L } \\
\text { M }\end{array}$ & $\begin{array}{l}\mathrm{P} \\
\mathrm{L} \\
\mathrm{M}\end{array}$ & $\begin{array}{l}P \\
V \\
D\end{array}$ & $\begin{array}{l}\text { A } \\
\text { V } \\
\text { B }\end{array}$ & $\begin{array}{l}\mathrm{P} \\
\mathrm{V} \\
\mathrm{C}\end{array}$ & $\begin{array}{l}\text { A } \\
\text { V } \\
\text { A }\end{array}$ & $\begin{array}{l}\text { A } \\
\text { V } \\
\text { D }\end{array}$ & $\begin{array}{l}\mathrm{A} \\
\mathrm{V} \\
\mathrm{M}\end{array}$ & $\begin{array}{l}\text { D } \\
\text { V } \\
\text { A }\end{array}$ \\
\hline 1 & 1 & -1 & -1 & -1 & 1 & 1 & 1 & -1 & & 26 & 1 & -1 & 1 & -1 & 1 & 1 & -1 & -1 & \\
\hline 2 & -1 & -1 & -1 & 1 & -1 & -1 & 1 & -1 & & 27 & -1 & -1 & -1 & 1 & 1 & 1 & -1 & -1 & \\
\hline 3 & -1 & -1 & -1 & 1 & -1 & 1 & 1 & -1 & & 28 & 1 & 1 & -1 & -1 & 1 & -1 & -1 & -1 & \\
\hline 4 & 1 & -1 & -1 & -1 & 1 & -1 & 1 & -1 & & 29 & -1 & 1 & -1 & 1 & 1 & -1 & 1 & 1 & \\
\hline 5 & 1 & -1 & -1 & -1 & 1 & 1 & -1 & -1 & & 30 & 1 & -1 & 1 & -1 & 1 & -1 & 1 & -1 & \\
\hline 6 & 1 & -1 & -1 & 1 & 1 & -1 & 1 & -1 & & 31 & -1 & -1 & -1 & -1 & 1 & -1 & -1 & -1 & \\
\hline 7 & -1 & -1 & -1 & 1 & 1 & -1 & -1 & -1 & & 32 & -1 & 1 & -1 & -1 & 1 & -1 & -1 & -1 & \\
\hline 8 & -1 & -1 & -1 & 1 & 1 & -1 & 1 & -1 & & 33 & 1 & -1 & 1 & 1 & 1 & 1 & -1 & -1 & \\
\hline 9 & 1 & -1 & 1 & 1 & 1 & 1 & 1 & -1 & & 34 & -1 & -1 & -1 & -1 & -1 & 1 & 1 & -1 & \\
\hline 10 & -1 & -1 & -1 & -1 & 1 & -1 & 1 & -1 & & 35 & 1 & -1 & -1 & 1 & 1 & -1 & -1 & -1 & \\
\hline 11 & -1 & -1 & -1 & -1 & 1 & 1 & -1 & -1 & & 36 & 1 & -1 & -1 & -1 & 1 & -1 & -1 & -1 & \\
\hline 12 & 1 & -1 & 1 & -1 & 1 & 1 & 1 & -1 & & 37 & -1 & 1 & 1 & -1 & 1 & -1 & 1 & -1 & \\
\hline 13 & -1 & -1 & -1 & 1 & 1 & 1 & 1 & -1 & & 38 & 1 & 1 & 1 & -1 & 1 & -1 & 1 & -1 & \\
\hline 14 & -1 & -1 & 1 & 1 & 1 & 1 & 1 & -1 & & 39 & -1 & 1 & -1 & -1 & 1 & 1 & 1 & 1 & \\
\hline 15 & -1 & -1 & 1 & 1 & 1 & -1 & 1 & -1 & & 40 & -1 & -1 & 1 & 1 & -1 & -1 & 1 & -1 & \\
\hline 16 & -1 & -1 & -1 & -1 & 1 & 1 & 1 & -1 & & 41 & 1 & 1 & 1 & -1 & 1 & 1 & -1 & -1 & \\
\hline 17 & -1 & -1 & 1 & -1 & 1 & 1 & 1 & -1 & & 42 & -1 & 1 & 1 & -1 & 1 & 1 & -1 & -1 & \\
\hline 18 & 1 & -1 & 1 & 1 & 1 & -1 & 1 & -1 & & 43 & -1 & -1 & 1 & 1 & 1 & -1 & -1 & -1 & \\
\hline 19 & 1 & -1 & -1 & 1 & 1 & 1 & 1 & -1 & & 44 & -1 & 1 & 1 & 1 & 1 & -1 & 1 & -1 & \\
\hline 20 & -1 & -1 & 1 & -1 & 1 & 1 & -1 & -1 & & 45 & -1 & 1 & 1 & -1 & 1 & 1 & 1 & -1 & \\
\hline 21 & -1 & -1 & 1 & 1 & 1 & 1 & -1 & -1 & & 46 & -1 & 1 & -1 & 1 & 1 & 1 & 1 & -1 & \\
\hline 22 & 1 & -1 & -1 & 1 & 1 & 1 & -1 & -1 & & 47 & -1 & -1 & 1 & -1 & 1 & -1 & -1 & -1 & \\
\hline 23 & -1 & -1 & -1 & -1 & -1 & -1 & 1 & -1 & & 48 & -1 & 1 & 1 & 1 & 1 & 1 & 1 & -1 & \\
\hline 24 & -1 & 1 & -1 & -1 & 1 & -1 & 1 & 1 & & 49 & 1 & 1 & 1 & 1 & 1 & -1 & 1 & -1 & \\
\hline 25 & -1 & -1 & 1 & -1 & 1 & -1 & 1 & -1 & & 50 & -1 & -1 & 1 & -1 & -1 & 1 & 1 & -1 & \\
\hline
\end{tabular}

Figure 4. Sample polarity configurations. The top 50 polarity configurations sorted according to error from experiment 1 are shown. This circuit did not include the DVA interneuron, and hence there were 256 possible configurations $\left(2^{6}\right)$ in the complete sorted list. Thus, the top $10 \%$ of the list reported in Table 3A consists of the top 26 polarity configurations shown in this figure. The PVD sensory neuron class was not externally stimulated during this run. A polarity consistent with that which resulted from statistical considerations is shown as a lightly shaded box; a polarity that is not consistent is shown in an unshaded box. No polarity predictions were made for the AVA or DVA neurons. These columns are darkly shaded. In this experiment, the tenth and sixteenth configurations are entirely consistent with the consensus configuration predicted in this report.

predictions for the polarities of seven of the nine cell classes that constitute the tap withdrawal circuit. The strength of the prediction for a given neuron was roughly correlated with the density of chemical connections that that neuron makes with other cells in the circuit (White et al., 1986; see Fig. 1). Because it was the reversal potential of the chemical connections that was the critical factor in determining the fitness measure, no prediction would have been expected for neurons that make sparse chemical connections. All of the predictions from each of the four experiments were consistent. For example, if a cell was predicted to be inhibitory in the first experiment, any further predictions that were made in either the same or subsequent experiments were also inhibitory. Because there were no disparities between the four experiments, a predicted polarity configuration based on consensus results was derived. This consensus configuration is shown in Figure 6. Because the polarity of two cells (DVA and AVA) were not reliably predicted at any level of significance, there are multiple configurations that are consistent with this consensus configuration.
For each experiment, the configurations were sorted according to their fitness. Figure 5 represents the frequency distribution of the fitness function from four experiments. Three points should be made regarding these distributions. First, each of these distributions was multimodal, suggesting that there were definable populations of configurations with good fitness. The frequency distributions were not evenly bisected, as would have been expected if a single neuron polarity was critical to determining circuit function; a specific polarity for multiple neurons was required for good fitness. Second, the subpopulation of configurations with the best fitness was roughly delimited by the $\alpha$ level (the fraction of configurations that were $1 \mathrm{SD}$ above the mean of the frequency distribution) in each of the four experiments. Finally, the mean of the two distributions that corresponded to experiments in which DVA and PVD were tonically activated (Fig. 5C,D) were both lower than the mean of those distributions that corresponded to experiments in which DVA and PVD were not explicitly stimulated, suggesting that this manipulation increased the overall fitness. On the other hand, the inclusion of DVA (Fig. 5B,D) did 

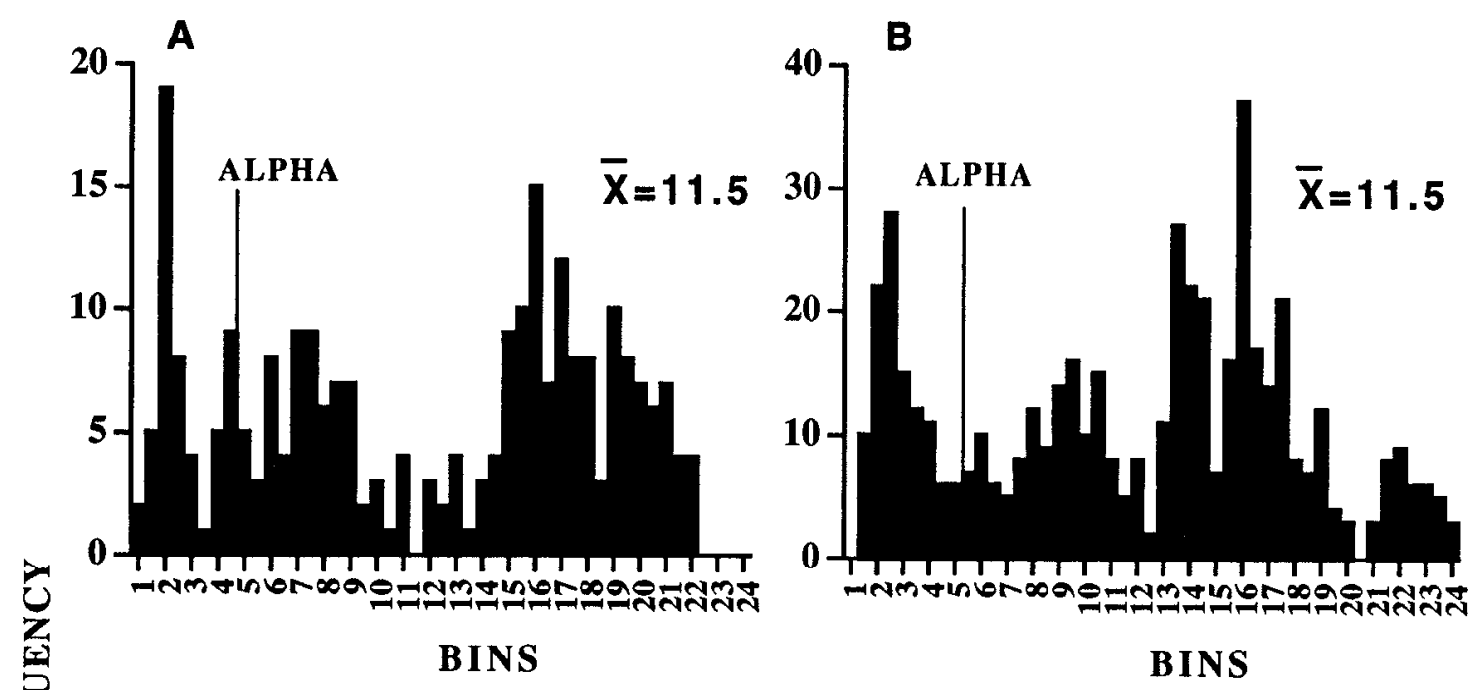

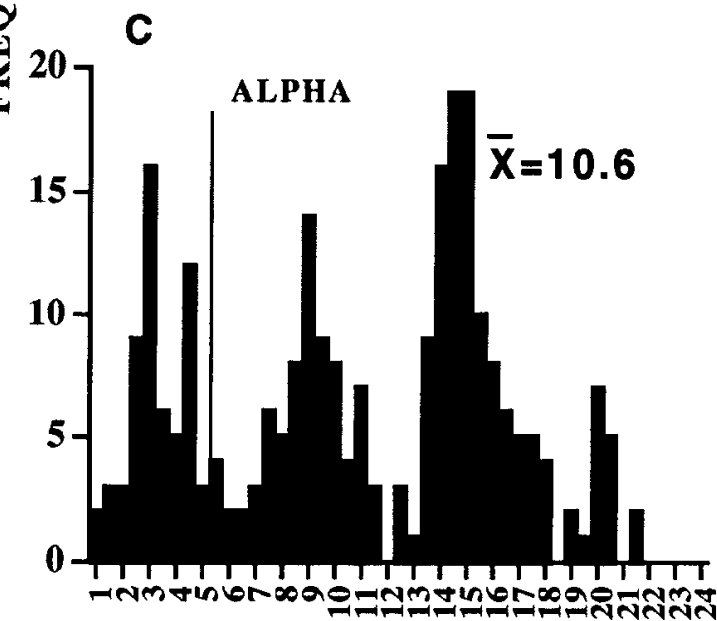

BINS

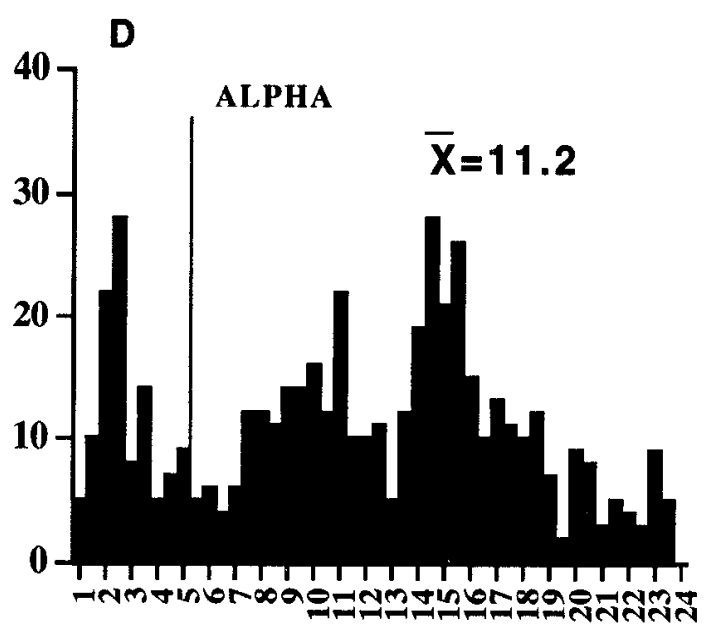

BINS

Figure 5. Fitness frequency distributions from four experinlents. The 256 possible configurations in experiments 1 and 3 and the 512 possible configurations from experiments 2 and 4 were sorted according to the fitness measure described in Materials and Methods. The $y$-axis represents the number of configurations with a given error in each of 24 error bins. The frequency distributions are multimodal. The fraction of the sorted list of configurations that corresponds to the $\alpha$ level (>1 SD below the mean of the distribution) lies to the left of the indicated $\alpha$ level in each case. The mean of each distribution is also indicated.

not systematically improve the fitness, as measured by the fitness distribution means.

\section{Experiment 1}

In the first experiment, the circuit was stimulated with a phasic "tap" to the mechanosensory neurons (PLM, ALM, and AVM) and was constructed without DVA. The best 50 polarity configurations from this run are presented in Figure 4. The results of this experiment are summarized in Table 3A. The three mechanosensory neurons (PLM, ALM, and AVM) were predicted to be functionally inhibitory, although the prediction for ALM was fairly weak. For example, significantly more polarity configurations in the top $10 \%$ (the top 26 configurations) of the list shown in Figure 4 possessed inhibitory polarities for the AVM neuron than possessed excitatory polarities for that cell. In contrast, the polarities of the AVA, AVB, and PVD neurons alternated with sufficiently high frequency in that same fraction of the list of configurations in Figure 4, that neither the excitatory nor inhibitory polarity could be said to predominate statistically for these cells. The two neurons (PVC and AVD) that couple the sensory input from the mechanosensory cells to the two neurons that control locomotion were both predicted to be excitatory.

\section{Experiment 2}

For the second experiment, DVA was added to the circuit and the same stimulation parameters were used. The results of this run are summarized in Table 3B. In this experiment, five of the nine cell classes possessed polarities that were correlated with good fit to behavioral data. The sensory neuron classes ALM and AVM were predicted to be inhibitory, although again, the ALM prediction was weak. Again, both of the neuron classes that modulate locomotion via mechanosensory input (PVC and AVD) were predicted to be excitatory (see Fig. 6). A prediction (inhibitory) was also made for the PVD cell class in this run. The DVA neuron class makes very sparse chemical connections with the rest of the circuitry, so no prediction for this cell was expected or obtained. In addition, no polarity predictions were made for the AVB or AVA neuron classes. 
Table 3. The results of a sign test analysis of the frequency of occurrence of a given polarity in various sections of the sorted list of all possible configurations for four experiments

\begin{tabular}{|c|c|c|c|c|c|}
\hline A Cell & Polarity? & $10 \%$ & $\alpha(22.7 \%)$ & $25 \%$ & $50 \%$ \\
\hline ALM & Inhibitory & $\mathrm{ns}$ & $p=0.0479$ & ns & $\mathrm{ns}$ \\
\hline PLM & Inhibitory & $p<0.0001$ & $p=0.0247$ & ns & $\mathrm{ns}$ \\
\hline AVM & Inhibitory & $p<0.0001$ & $p<0.0001$ & $p<0.0001$ & $p<0.0001$ \\
\hline PVD & $?$ & $\mathrm{~ns}$ & $\mathrm{~ns}$ & ns & ns \\
\hline $\mathrm{AVB}$ & $?$ & ns & ns & ns & ns \\
\hline PVC & Excitatory & $p<0.0001$ & $p<0.0001$ & $p<0.0001$ & $p<0.0001$ \\
\hline AVA & $?$ & ns & ns & ns & ns \\
\hline AVD & Excitatory & $p=0.0290$ & $p<0.0119$ & $p=0.0169$ & $p<0.0001$ \\
\hline B Cell & Polarity? & $10 \%$ & $\alpha(21.9 \%)$ & $25 \%$ & $50 \%$ \\
\hline ALM & Inhibitory & $\mathrm{ns}$ & ns & $p=0.0421$ & ns \\
\hline PLM & $?$ & ns & ns & $\mathrm{ns}$ & ns \\
\hline AVM & Inhibitory & $p<0.0001$ & $p<0.0001$ & $p<0.0001$ & $p<0.0001$ \\
\hline PVD & Inhibitory & $p<0.0001$ & $p=0.0153$ & $p=0.0011$ & $p=0.0208$ \\
\hline AVB & $?$ & ns & ns & ns & ns \\
\hline PVC & Excitatory & $p=0.0002$ & $p<0.0001$ & $p<0.0001$ & $p<0.0001$ \\
\hline AVA & $?$ & ns & $\mathrm{ns}$ & ns & ns \\
\hline AVD & Excitatory & $p<0.0001$ & $p<0.0001$ & $p<0.0001$ & $p<0.0001$ \\
\hline DVA & $?$ & ns & $\mathrm{ns}$ & ns & ns \\
\hline C Cell & Polarity? & $10 \%$ & $\alpha(19.9 \%)$ & $25 \%$ & $50 \%$ \\
\hline ALM & $?$ & ns & $\mathrm{ns}$ & ns & ns \\
\hline PLM & Inhibitory & $p=0.0094$ & $\mathrm{~ns}$ & $\mathrm{~ns}$ & $\mathrm{~ns}$ \\
\hline AVM & Inhibitory & $p<0.0001$ & $p<0.0001$ & $p<0.0001$ & $p<0.0001$ \\
\hline PVD & Inhibitory & ns & $p=0.0007$ & $p=0.0016$ & ns \\
\hline AVB & $?$ & $\mathrm{~ns}$ & $\mathrm{~ns}$ & ns & ns \\
\hline PVC & Excitatory & $p<0.0001$ & $p<0.0001$ & $p<0.0001$ & $p=0.0035$ \\
\hline AVA & $?$ & $\mathrm{~ns}$ & $\mathrm{~ns}$ & $\mathrm{~ns}$ & ns \\
\hline AVD & Excitatory & $\mathrm{ns}$ & $p=0.0003$ & $p=0.0002$ & $p=0.0011$ \\
\hline D Cell & Polarity? & $10 \%$ & $\alpha(21.7 \%)$ & $25 \%$ & $50 \%$ \\
\hline ALM & Inhibitory & $\mathrm{ns}$ & $p=0.0472$ & $p=0.0271$ & ns \\
\hline PLM & Inhibitory & $p=0.0018$ & ns & $\mathrm{ns}$ & ns \\
\hline AVM & Inhibitory & $p<0.0001$ & $p<0.0001$ & $p<0.0001$ & $p<0.0001$ \\
\hline PVD & $?$ & ns & ns & ns & ns \\
\hline AVB & Inhibitory & $p=0.0110$ & $\mathrm{~ns}$ & $\mathrm{~ns}$ & ns \\
\hline PVC & Excitatory & $p<0.0001$ & $p<0.0001$ & $p<0.0001$ & $p=0.0014$ \\
\hline AVA & $?$ & ns & $\mathrm{ns}$ & ns & ns \\
\hline AVD & Excitatory & $p=0.0002$ & $p<0.0001$ & $p<0.0001$ & $p<0.0001$ \\
\hline DVA & $?$ & ns & $\mathrm{ns}$ & ns & ns \\
\hline
\end{tabular}

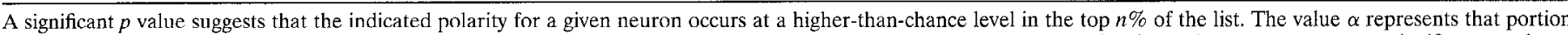

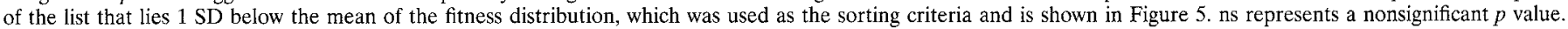

\section{The addition of tonic activation of PVD and DVA}

The PVD cell class probably does not directly detect the tap stimulus; in the absence of AVM, ALM, and PLM, worms do not respond to tap even if PVD is left intact (Chalfie and Sulston, 1981; Wicks and Rankin, 1995a). Rather, its morphology and the behavior of animals that lack this cell suggest that it may act as a stretch receptor (Ed Hedgecock, ciled in Way and Chalfie, 1988). It is also possible that the cell responds to some interoceptive cue, which makes the animal more or less responsive in accord with the level of stimulation in its environment, analogous to the dynamic gain control system described by Fischer and Carew (1993). In either case, the cell may provide tonic input to the tap withdrawal circuitry rather than the phasic input provided by the tap. Similarly, the DVA interneuron that receives heavy input from cells that carry chemosensory information may modulate the animal's responsiveness according to the nature of the chemical environment (Dusenbury, 1974). This kind of input, like the proposed PVD input described above, was modeled by applying a low tonic stimulation of the DVA interneuron in situ. Thus, two additional experiments were run, which were identical to the first two except that the PVD and DVA cell classes received low tonic depolarization throughout the duration of the simulations.

\section{Experiment 3}

In the first of these two experiments, the circuit was constructed without DVA, and PVD was tonically activated at one-quarter the intensity of the phasic tap stimulus. The results of this run are shown in Table 3C. This experiment can be directly compared with experiment 1 to assess the impact of tonic stimulation. The main difference between these two results is that when the PVD 


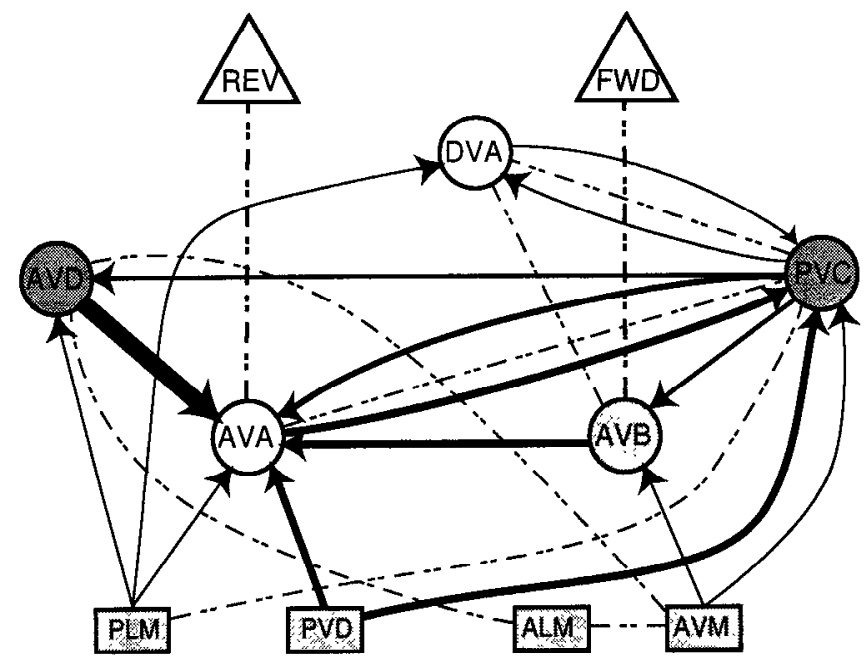

Figure 6. Simplified circuit with predicted polarities. The circuit that mediates the nematode tap withdrawal reflex consists of seven sensory neurons (squares), nine interneurons (circles), and two motorneuron pools (not shown), which produce forward and backward locomotion (triangles). All cells represent bilateral classes of cells except AVM and DVA, which are single cells. Chemical connections are indicated by arrows, with the number of synaptic contacts proportional to the width of the arrow. Gap junctions are indicated by dotted lines. This circuit has been simplified for ease of presentation in two ways: the bilateral symmetry of the circuit has been collapsed, and only classes of connections with an average of greater than five synaptic contacts are shown. The consensus polarities of the neurons in this circuit, which were derived from four experiments, are also shown. Neurons that are predicted to make excitatory connections are darkly shaded, whereas neurons that are predicted to make inhibitory connections are lightly shaded. Two neurons (AVA and DVA) did not possess polarities that clustered at above chance levels in any of the experiments presented in this report.

cell class was activated, its polarity became more highly correlated with a good fit to the behavioral results with a concurrent decrease in the correlation of an inhibitory polarity for the mechanosensory neurons PLM and ALM. The predictions concerning polarities for PVC, AVD, and AVM were maintained from experiment 1 to experiment 3 .

\section{Experiment 4}

In the last experiment, the modeled circuit was stimulated by a phasic input to the mechanosensory neurons (PLM, ALM, AVM) and a tonic input to both the DVA and the PVD neurons, and all possible polarity configurations were assessed. The results of each of the one-sample sign tests used to assess potential clustering of a specific polarity are shown in Table 3D. The three mechanosensory neurons that were stimulated in this experiment (PLM, ALM, AVM) were predicted to be inhibitory. Of the six other cell classes tested in this run, a prediction was made for the polarities of three of them. Of the two neurons that constitute the gearbox (AVA and AVB), AVB was predicted to be inhibitory. The two neurons (AVD and PVC) that connect much of the mechanosensory input to the driver neurons were again predicted to be excitatory. No prediction was made for either the DVA neuron or the PVD neuron. It is somewhat surprising that no prediction was made for PVD in this run. Given that experiment 3 suggested that tonic activation of PVD increased the correlation between an inhibitory polarity for that cell and a good fit to the behavioral data, it was expected that this relationship would be maintained in experiment 4 because in this experiment, PVD was also tonically stimulated. However, there appears to be an interaction between the way PVD is stimulated and the presence of DVA.

These results can be contrasted with those from experiment 3 to again assess the impact of the addition of DVA to the mechanosensory integration circuitry. As in the first two experiments, the addition of DVA to the circuitry had several effects. First, it increased the correlation between the polarities of two cell classes and a good fit to behavioral data; ALM and AVB did not possess significant polarities in experiment 3 , but did in experiment 4 . A determined polarity for one cell class (PVD) became less correlated with a good fit to behavioral data. Again, this result is surprising, because in the first two experiments PVD was predicted to be inhibitory only when DVA was present.

The results of experiment 4 were compared with those from the second experiment to allow an assessment of the effect of tonic stimulation with DVA in the circuit. There was a decrease in the significance of the polarity of PVD relative to run two as a result of tonic activation of that neuron. However, the main difference between these two runs was that AVB was predicted to be inhibitory in the last run but not in the second. Thus, the addition of tonic stimulation of PVD and DVA increases the relative significance of the polarity of AVB.

Overall, the strongest set of predictions was made for the AVM, PVC, and AVD cell classes. The AVD class makes among the most dense set of chemical synaptic connections within the animal (White et al., 1986; Achacoso and Yamamoto, 1992) (see Fig. 6); therefore, its predicted polarity would be expected to be important in the determination of tap withdrawal circuit behavior. Similarly, the AVM class makes most of the chemical connections from the anterior mechanosensory field of the animal (White et al., 1986), and thus its polarity would also be expected to be critical in shaping an animal's response to a tap stimulus.

Finally, because polarity configurations that were consistent with the consensus configuration appeared several times in each experiment (attributable to redundancy of the polarities of neurons that did not achieve significance; see Fig. 4), a mean consensus profile was constructed for each of the experiments. These mean consensus profiles (Fig. 7) produced remarkably similar fits to the target profile. Regardless of the stimulation parameters tested or whether DVA was present in the circuit, these results all differed from the target profile in two consistent ways. First, under all four experimental conditions, the relative effects of ablation of the sensory neurons on the behavior of the model did not correspond to that observed in vivo. That is, the ablation of AVM in the model produced a larger relative change in behavior than was observed in the worm, whereas the ablation of PLM in the model produced a smaller relative change in behavior than was observed in the worm. It was an assumption in these experiments that the transduction efficiency of all of the sensory neurons was the same; however, it is likely that this is not the case. The sensory processes of the PLM neurons are situated on the lateral aspects of the worm, whereas the AVM process runs in the ventral aspect of the worm (Chalfie and Sulston, 1981), and therefore may be differentially activated by a tap stimulus. Second, the ablation of PVD in the animal resulted in a significant decrease in the magnitude of the withdrawal reflex. In all of the best fit configurations, including the consensus configuration, the ablation of PVD had little effect on the output of the modeled circuit.

\section{The effect of varying synaptic conductance}

Two physiological parameters (the gap junction conductance, $\hat{g}$, and the maximal synaptic conductance, $\bar{g}$ ), were each varied over 


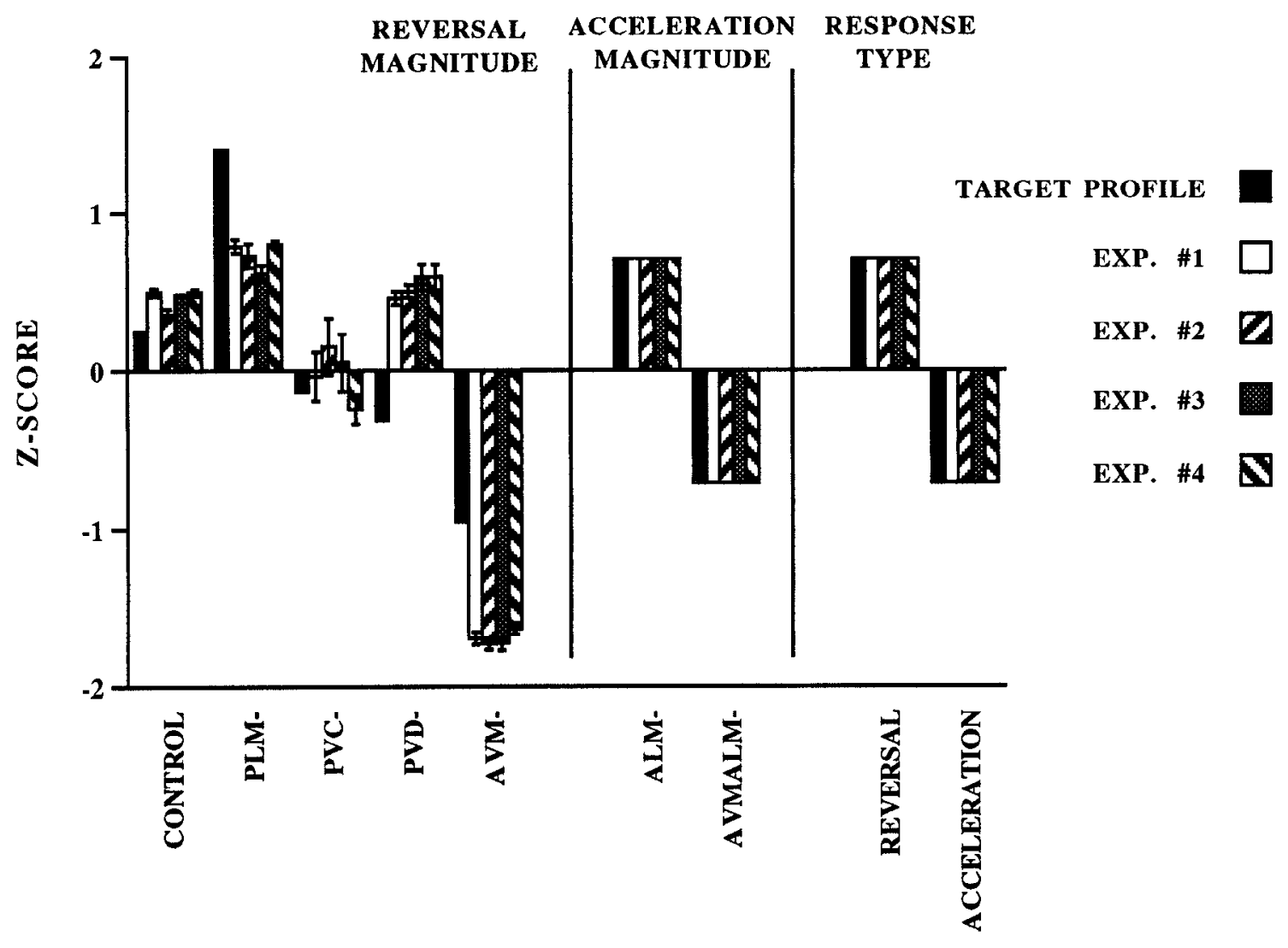

Figure 7. Mean consensus configuration fit. The best fit profiles from four experiments are shown in comparison to the empirical data to which they were compared for those configurations that were consistent with the consensus configuration in Figure 6 . These data sets are each expressed as 7-scores around the mean of that data set, because only changes relative to the intact condition are interpretable. All four simulated data sets differ from the intact condition in two consistent ways. First, the PVD ablation had a large effect on the reversal magnitude of real animals, but had little effect on the modeled response. Second, the relative effects of touch-cell ablations in the model are not consistent with the changes produced by ablations in worms. Specifically, the relative effects of the AVM and PLM ablations are reversed in the model as compared with the worm. This may be attributable to mechanical processes that affect the transduction efficiencies of these touch cells. Error bars indicate SEM.

two orders of magnitude to confirm that the exact values of these parameters were not critical in the prediction of neuron polarities. Experiment 1 was replicated under four distinct parameter conditions. In experiments 5 and 7, the gap junction conductance was increased $\left(\hat{g}=5 \times 10^{-8} \mathrm{~S}\right)$ and decreased $\left(\hat{g}=5 \times 10^{-10} \mathrm{~S}\right)$, respectively, from the value used in experiment 1 . In experiments 6 and 8 , the synaptic conductance was increased $\left(\bar{g}=6 \times 10^{-9} \mathrm{~S}\right)$ and decreased $\left(\bar{g}=6 \times 10^{-11} \mathrm{~S}\right)$, respectively, from the value used in experiment 1 . The results of these experiments are presented in Table 4. Although the number of predictions made in these further experiments was variable, there were no contradictions between these results and those reported earlier. In general, any manipulation that decreased the ratio of $\hat{g}$ to $\bar{g}$ decreased the number of predictions made by the model. Although these manipulations certainly did have effects both on the absolute values of the propensity to reverse of any given polarity configuration and on the exact order of polarity configurations, the strategy used to predict neuron polarities appeared to be relatively insensitive to the exact parameters used in the simulation. This is consistent with preliminary versions of these experiments, in which neurons were modeled as electrical circuits and synapses as entirely linear entities. Under these conditions-in which parameters were not even physiologically motivated, let alone justified-the strategy used to form predictions concerning synaptic character yielded the same set of predictions as are presented here.

\section{DISCUSSION}

The use of a simple computational model of the nematode tap withdrawal circuit has allowed the prediction of an array of synaptic polarities of the neurons that compose that circuit. The electrophysiological intractability of the worm's nervous system

\section{Table 4. The effects of varying the values of the maximal synaptic conductance $(\overline{\mathrm{g}})$ and the gap conductance $(\hat{\mathrm{g}})$ over three orders of magnitude on polarity predictions}

\begin{tabular}{lcccccc} 
Cell & Exp. 1 & Exp. 5 & Exp. 6 & Exp. 7 & Exp. 8 & Consensus \\
\hline ALM & Inhibitory & Inhibitory & $?$ & $?$ & $?$ & Inhibitory \\
PLM & Inhibitory & $?$ & $?$ & $?$ & $?$ & Inhibitory \\
AVM & Inhibitory & Inhibitory & Inhibitory & Inhibitory & Inhibitory & Inhibitory \\
PVD & $?$ & $?$ & $?$ & $?$ & Inhibitory & Inhibitory \\
AVB & $?$ & Inhibitory & $?$ & $?$ & $?$ & Inhibitory \\
PVC & Excitatory & Excitatory & Excitatory & Excitatory & Excitatory & Excitatory \\
AVA & $?$ & $?$ & $?$ & $?$ & $?$ & $?$ \\
AVD & Excitatory & Excitatory & $?$ & $?$ & Excitatory & Excitatory \\
DVA & $?$ & $?$ & $?$ & $?$ & $?$ & $?$ \\
\hline
\end{tabular}

Experiment 1 was replicated with the gap junction conductance increased (experiment 5) or decreased (experiment 7) an order of magnitude or with the synaptic conductance increased (experiment 6) or decreased (experiment 8) an order of magnitude. Although the number of predictions varied, no changes in the consensus configuration were suggested as a consequence of these manipulations. 
has previously made it very difficult to address this problem. However, the availability of data sets describing the behavior of animals with lesions in the tap withdrawal reflex circuitry (Wicks and Rankin, 1995a) suggested a novel approach. A model of the circuit was produced, and lesions in the modeled circuit that were isomorphic to those produced in vivo were made. The behavior of real animals was then used as a target to which the output of the modeled circuit was optimized by altering the array of underlying polarities within the model. To our knowledge, this approach has not been used before.

In addition to predicting polarities for seven of the nine cells in the circuit, experiments were run to assess the impact of two other variables: (1) the tonic stimulation of DVA and PVD; and (2) the removal of DVA from the mechanosensory integration circuitry. These tests provide some support for the tonic stimulation of PVD and DVA by background mechanosensory and chemosensory cues; less discrepancy between the model and behavior of worms was observed in runs that included low tonic stimulation of these cells. In addition, a prediction for the polarity of AVB was made only in a run that included tonic stimulation. The removal of DVA neither systematically improved the model's fit to behavioral data nor resulted in more polarity predictions for tap withdrawal circuit neurons.

\section{Sensory neurons}

The first generalization that can be drawn from the results of these experiments is that neurons naturally cluster according to the array of predicted polarities into groups that correspond to anatomically or genetically defined classes. The sensory neurons modeled in this report fall into two broad classes: the touch cells [PLM, AVM, and ALM (Chalfie and Sulston, 1981)] and the putative stretch receptor PVD. The touch cells are a genetically distinct group of neurons. They express an array of active promoters, which result in the production of a distinct neuronal morphology that is crucial to the mechanosensory function of these neurons (Chalfie and Sulston, 1981; Way and Chalfie, 1988; Savage et al., 1989). Given that these cells share a common genetic program, it might also be expected that they share a common neurotransmitter phenotype. The results presented here are consistent with this speculation; all three of these cells were predicted to be inhibitory.

It has been hypothesized that the role of the chemical connections from these sensory neurons onto the interneurons is to functionally inhibit the inappropriate response (Chalfie et al., 1985; Rankin, 1991; Wicks and Rankin, 1995a,b). For example, if the PLM sensory neurons were strongly activated, then it would promote forward locomotion by exciting PVC (and consequently AVB; see Fig. 6) via coupling to that cell, as well as simultaneously reduce backward locomotion via the inhibition of AVD and AVA. Behaviorally, a key distinction between the tap response and the touch response is that the tap stimulus activates both the anterior and posterior touch cells concurrently, making the behavioral output of the circuit a balance between the relative levels of activation within two subcircuits (Wicks and Rankin, 1995a,b). The functional inhibition of antagonistic subcomponents of behavior is a critical and potentially informative aspect of the tap withdrawal circuit function.

\section{Interneurons}

The two interneurons (AVD and PVC) that were predicted to be excitatory also fall into an intuitive functional category first proposed by Chalfie et al. (1985). These neurons each couple elec- trical input from the appropriate sensory neuron field onto the interneurons that drive locomotion. Thus, AVD receives electrical input from the animal's anterior mechanosensory field and relays that information onto the AVA interneuron to drive backward locomotion. The polarity of the connection between AVD and AVA was predicted to be excitatory, which is consistent with this hypothesized role. Similarly, the connection between PVC and AVB was also predicted to be excitatory.

Of the two interneurons that drive the motor neurons responsible for locomotion (AVA and AVB), one was predicted to be inhibitory. This predicted polarity might appear to be inconsistent with the role that these cells appear to play in circuit functionthe excitation of locomotion. However, because gap junctions from these neurons onto motor neurons in the ventral cord presumably mediate that excitation, the predicted inhibitory phenotype of these neurons would not contradict that role. Again, these two cells each appear to perform the same function for the two subcircuits of the tap withdrawal reflex-one mediating forward locomotion and one mediating backward locomotion-and hence form a functional class. Although in these experiments no prediction for the polarity of AVA was made, we would predict, based on a conservation of class function, that AVA is more likely to be inhibitory than excitatory.

The PVD sensory neuron was predicted to be inhibitory whether it was tonically activated during the simulation or not (although no prediction was made for PVD if it was tonically activated and DVA was included in the circuit; see Table 3). The cell configuration of PVD and its two postsynaptic partners (PVC and AVA) may act as a dynamic gain control for the tap withdrawal circuit in the same way as described for the L30-L29 interneuron pair (and the synaptic input to these neurons from the siphon sensory neurons) in the Aplysia siphon withdrawal reflex (Blazis et al., 1993; Fischer and Carew, 1993). In essence, these cells may be sensitive to the amount of mechanosensory stimulation that the animal experiences and modulate the animal's response accordingly. In $C$. elegans, PVD may provide the input to this functional subcircuit. If AVA may be considered inhibitory, then this motif-an inhibitory and an excitatory cell each connected by both electrical and chemical connections and each receiving input from the same sensory source-may represent a general biological solution for gain control by a small neural subcircuit.

The polarity of the interneuron DVA was not explicitly predicted by this model presumably because this neuron makes few chemical connections within the circuit. However, the cell does make significant electrical connections with both PVC and AVB, and therefore may play a role in the integration of a tap stimulus. Although the largest number of polarity predictions was made in experiment 4 when DVA was present, the best fit to behavioral data (experiment 3 ) as gauged by the mean of the fitness distribution was observed when DVA was removed from the circuit. Thus, we are unable to confirm a role for DVA based on these results. A more thorough treatment of the possible chemosensory role of DVA in the modulation of locomotion might explicitly incorporate the chemosensory input (Bargmann and Horvitz, 1991) and more formally model chemosensory gradients, perhaps incorporating the methods of Lockery et al. (1993).

Although a single neurotransmitter may have both an excitatory and an inhibitory effect postsynaptically, depending on what receptor that postsynaptic partner expresses at that synapse, these experiments assume that this is a rare occurrence. There is, however, evidence in $C$. elegans of at least one example of a single 
neurotransmitter having both an excitatory and an inhibitory effect. The neurotransmitter GABA has been shown to have both functionally excitatory and inhibitory effects in the worm (McIntire et al., 1993). To investigate this possibility, the polarity of all synaptic classes from a single neuron would have to be allowed to vary independently of all others. Furthermore, each synaptic class could be allowed to assume one of multiple reversal potentials, or specifically increase or decrease postsynaptic conductance (Getting, 1989). These changes might shed light on the function of synaptic classes, which remains unclear even in light of these results. Chalfie et al. (1985) point out that it is difficult to intuit a role for a number of touch circuit synapses (for example, the PVC to AVD class) and suggest that such classes may prove critical in determining the timing of sensorimotor integration. This hypothesis could bcar further cxamination using the method presented here. These changes, along with a more complete investigation of the effects of varying the physiological parameters in this model, could be implemented by adopting a more relaxed mechanism to search a larger parameter space, such as a genetic algorithm.

Many neurotransmitter types have been identified in C. elegans, including GABA (McIntire et al., 1993), dopamine (Sulston et al., 1975), serotonin (Horvitz et al., 1982; Loer and Kerlyon, 1994), octopamine (Horvitz et al., 1982), glutamate (Arena et al., 1992), and acetylcholine (Chalfie and White, 1988). However, the neurotransmitter receptor pairings of the nematode tap withdrawal circuit neurons are as yet unknown. The predictions made in this report, we hope, will facilitate the identification of putative neurotransmitters in this circuit. Furthermore, the method used to make these predictions may be generalized to determine properties of other neural circuits that mediate behaviors modulated by lesion.

\section{REFERENCES}

Achacoso TB, Yamamoto WS (1992) AY's neuroanatomy of C. elegans for computation. Boca Raton, FL: CRC.

Arena JP, Liu KK, Paress PS, Schaeffer JM, Cully DF (1992) Expression of a glutamate-activated chloride current in Xenopus oocytes injected with Caenorhabditis elegans RNA: evidence for modulation by avermectin. Mol Brain Res 15:339-348.

Avery L, Horvitz HR (1987) A cell that dies during wild-type C. elegans development can function as a neuron in a ced-3 mutant. Cell 51:1071-1078.

Avery L, Horvitz HR (1989) Pharyngeal pumping continues after laser kiling of the pharyngeal nervous system of $C$. clegans. Neuron 3:473-485.

Avery L, Raizen D, Lockery S (1995) Electrophysiological methods. In: Caenorhabditis elegans: modern biological analysis of an organism (Epstein HF, Shakes DC, eds), pp 251-269. San Diego: Academic.

Bargmann CI, Horvitz HR (1991) Chemosensory neurons with overlapping functions direct chemotaxis to multiple chemicals in C. elegans. Neuron 7:729-742.

Bennett MVL (1972) A comparison of electrically and chemically mediated transmission. In: Structure and function of synapses (Pappas GD, Purpura DP, eds), pp 221-256. New York: Raven.

Blazis DEJ, Fischer TM, Carew TJ (1993) A neural network model of inhibitory information processing in Aplysia. Neural Comput 5:213-227.

Brenner S (1974) The genetics of the nematode Caenorhabditis elegans. Genctics 77:71-94.

Carew TJ, Pinsker HM, Kandel ER (1972) Long-term habituation of a defensive reflex in Aplysia. Science 175:451-454.

Chalfie M, Sulston J (1981) Developmental genetics of the mechanosensory neurons of Caenorhabditis elegans. Dev Biol 82:358-370.

Chalfie M, White JG (1988) The nervous system. In: The nematode Caenorhabditis elegans (Wood WB, ed), pp 337-392. Cold Spring Harbor, NY: Cold Spring Harbor.

Chalfie M, Sulston JE, White JG, Southgate E, Thomson JN, Brenner S (1985) The neural circuit for touch sensitivity in Caenorhabditis elegans. J Neurosci 5:956-964.
Corfas G, Dudai Y (1990) Adaptation and fatigue of a mechanosensory neuron in wild-type Drosophila and in memory mutants. J Neurosci 10:491-499.

Davis RE, Stretton AOW (1989a) Passive membrane properties of motorneurons and their role in long-distance signaling in the nematode Ascaris. J Neurosci 9:403-414.

Davis RE, Stretton AOW (1989b) Signaling properties of Ascaris motorneurons: graded active responses, graded synaptic transmission, and tonic transmitter release. J Neurosci 9:415-425.

DeSchutter E, Angstadt JD, Calabrese RL (1993) A model of graded synaptic transmission for use in dynamic network simulations. J Neurophysiol 69:1225-1235.

du Lac S, Raymond JL, Sejnowski TJ, Lisberger SG (1995) Learning and memory in the vestibulo-ocular reflex. Annu Rev Neurosci 18:409-441.

Dusenbury DB (1974) Analysis of chemotaxis in the nematode Caenorhabditis elegans by countercurrent separation. J Exp Zool 188:41-47.

Erdös P, Niebur E (1993) Theory of locomotion of nematodes: control of the somatic motor neurons by interneurons. Math Biosci 118:51-82.

Fischer TM, Carew TJ (1993) Activity-dependent regulation of recurrent inhibition: a mechanism for dynamic gain control in the siphon withdrawal reflex of Aplysia. J Neurosci 13:1302-1314.

Getting PA (1989) Emerging principles governing the operation of neural networks. Annu Rev Neurosci 12:185-204.

Gross RE, Bagchi S, Lu X, Rubin CS (1990) Cloning, characterization, and expression of the gene for the catalytic subunit of cAMP-dependent protein kinase in Cacnorhabditis elegans. J Biol Chem 265:6896 6907.

Groves PM, De Marco R, Thompson RF (1969) Habituation and sensitization of spinal interneuron activity in acute spinal cat. Brain Res 14:521-525.

Hall DH, Russell RL (1991) The posterior nervous system of the nematode Caenorhabditis elegans: serial reconstruction of identified neurons and complete pattern of synaptic interactions. J Neurosci 11:1-22.

Harris-Warrick RM, Nagy F, Nusbaum MP (1992) Neuromodulation of stomatogastric networks by identified neurons and neurotransmitters. In: Dynamic ncural networks (Harris-Warrick RM, Marder E, Selverston AI, Moulins M, eds), pp 87-137. Cambridge, MA: MIT.

Ilorvitz IIR, Chalfie M, Trent C, Sulston JE, Evans PD (1982) Scrotonin and octopamine in the nematode $C$. elegans. Science 216:1012-1014.

Kandel ER, Schwartz JH (1982) Molecular biology of learning: modulation of transmitter release. Science 218:433-442.

Krasne FB (1969) Excitation of habituation of the crayfish escape reflex: the depolarizing response in lateral giant fibers in the isolated abdomen. J Exp Biol 50:29-46.

Lockery SR, Sejnowski TJ (1992) Distributed processing of sensory information in the leech. III. A dynamic neural network of the local bending reflex. J Neurosci 12:3877-3895.

Lockery SR, Nolan SJ, Sejnowski TJ (1993) Modeling chemotaxis in the nemlatode $C$. elegans. In: Computation and neural systems (Eeckman FH, Bower JM, eds), pp 249-254. Boston: Kluwer Academic.

Loer CM, Kenyon CJ (1994) Serotonin-deficient mutants and male mating-behavior in the nematode Caenorhabditis elegans. J Neurosci 13:5407-5417.

Lu X, Gross RE, Bagchi S, Rubin CS (1990) Cloning, structure, and expression of the gene for a novel regulatory subunit of cAMPdependent protein kinase in Caenorhabditis elegans. J Biol Chem 265:3293-3303.

McIntire SL, Jorgensen E, Kaplan J, Horvitz HR (1993) The GABAergic nervous system of Caenorhabditis elegans. Nature 364:337-341.

Mendel JE, Korswagen HC, Liu KS, Hajdu-Cronin YM, Simon MI, Plasterk RHA, Sternberg PW (1995) Participation of the protein G(o) in multiple aspects of behavior in C. elegans. Science 167:1652-1655.

Niebur E (1988) Théorie du système locomoteur et neuronal des nématodes. PhD thesis, University of Lausanne.

Niebur E, Erdös P (1991) Theory of the locomotion of nematodes: dynamics of undulatory progression on a surface. Biophys J 60:1132-1146.

Niebur E, Erdös P (1993) Theory of the locomotion of nematodes: control of the somatic motor neurons by interneurons. Math Biosci 118:51-82.

Nonet ML, Grundahl K, Meyer BJ, Rand JB (1993) Synaptic function is impaired but not eliminated in C. elegans mutants lacking synaptotagmin. Cell 73:1291-1305.

Press WH, Flannery BP, Teukolsky SA, Vetterling WT (1988) Numerical recipes in $\mathrm{C}$ : the art of scientific computing. Cambridge, UK: Cambridge UP. 
Raizen DM, Avery L (1994) Electrical activity and behavior in the pharynx of Caenorhabditis elegans. Neuron 12:483-495.

Rall WB (1977) Core conductor theory and cable properties of neurons. In: Handbook of physiology: the nervous system, Vol 1 (Kandel ER, ed), pp 39-97. Bethesda, MD: American Physiological Society.

Rall WB (1989) Cable theory for dendritic neurons. In: Methods in neuronal modeling: from synapses to networks (Kock $C$, Segev 1 , eds), pp 9-62. Cambridge MA: MIT.

Rankin CH (1991) Interactions between two antagonistic reflexes in the nematode Caenorhabditis elegans. J Comp Physiol [A] 169:59-67.

Rankin CH, Beck CDO, Chiba CM (1990) Caenorhabditis elegans: a new model system for the study of learning and memory. Behav Brain Res 37:89-92.

Roehrig C, Wicks SR, Rankin CH (1994) Determination of synaptic polarities using a computational cellular model of the nematode tap withdrawal reflex. Soc Neurosci Abstr 20:1201.

Savage C, Hamelin M, Culotti JG, Coulson A, Albertson DG, Chalfie M (1989) mec-7 is a beta-tubulin gene required for the production of 15-protofilament microtubules in Caenorhabditis elegans. Genes Dev 3:870-881.

Segalat L, Elkes DA, Kaplan JM (1995) Modulation of serotonincontrolled behaviors by $\mathrm{G}(\mathrm{o})$ in Caenorhabditis elegans. Science 267:1648-1651.

Segev I, Fleshman JW, Burke RE (1989) Compartmental models of complex neurons. In: Methods in neuronal modeling: from synapses to networks (Kock C, Segev I, eds), pp 63-96. Cambridge, MA:MIT.

Selverston AI (1985) Model neural networks and behavior. New York: Plenum.

Sherrington $C(1906)$ The integrative action of the nervous system. New Haven, CT: Yale UP.

Siegal S (1956) Nonparametric statistics for the behavioral sciences. New York: McGraw-Hill.

Steinmetz JE, Lavond DG, Ivkovich D, Logan CG, Thompson RF (1992) Disruption of classical eyelid conditioning after cerebellar lesions: damage to a memory trace system or a simple performance deficit. J Neurosci 12:4403-4426.
Stern MJ, DeVore DL (1994) Extending and connecting signaling pathways in C. elegans. Dev Biol 166:443-459.

Stretton A, Donmoyer J, Davis R, Meade J, Cowden C, Sithigorngul P (1992) Motor behavior and motor nervous system function in the nematode Ascaris suum. J Parasitol 78:206-214.

Sulston JE, White JG (1980) Regulation and cell autonomy during postembryonic development of Caenorhabditis elegans. Dev Biol 78:577-597.

Sulston JE, Dew M, Brenner S (1975) Dopaminergic neurons in the nematode Caenorhabditis elegans. J Comp Ncurol 163:215-226.

Vu ET, Lee SC, Krasne FB (1993) The mechanism of tonic inhibition of crayfish escape behavior: distal inhibition and its functional significance. J Neurosci 13:4379-4393.

Ward S, Thomson N, White JG, Brenner S (1975) Electron microscopical reconstruction of the anterior sensory anatomy of the nematode Caenorhabditis elegans. J Comp Neurol 160:313-337.

Ware RW, Clark D, Crossland K, Russel RL (1975) The nerve ring of the ncmatode Caenorhabditis elegans: sensory input and motor output. J Comp Neurol 162:71-110.

Way JC, Chalfie M (1988) mec-3, a homeobox-containing gene that specifies differentiation of the touch receptor neurons in C. elegans. Cell 54:5-16.

White JG, Southgate E, Thomson JN, Brenner S (1976) The structure of the ventral cord of Caenorhabditis elegans. Philos Trans R Soc Lond Biol 275:327-384.

White JE, Southgate E, Thomson JN, Brenner S (1986) The structure of the nervous system of the nematode Caenorhabditis elegans. Philos Trans R Soc Lond Biol 314:1-340.

Wicks SR, Rankin CH (1995a) Integration of mechanosensory stimuli in Caenorhabditis elegans. J Neurosci 15:2434-2444.

Wicks SR, Rankin CH (1995b) Recovery from habituation and habituation of spontaneous behavior in $C$. elegans: dependence on circuit characteristics. Soc Neurosci Abstr 21:1201.

Wood WB (1988) The nematode Caenorhabditis elegans. Cold Spring Harbor, NY: Cold Spring Harbor. 